\title{
Monosynaptic Hippocampal-Prefrontal Projections Contribute to Spatial Memory Consolidation in Mice
}

\author{
Sonja Binder, ${ }^{1,2}$ Matthias Mölle, ${ }^{2}$ Michael Lippert, ${ }^{4}$ Ralf Bruder, ${ }^{3}$ Sonat Aksamaz, ${ }^{1}$ Frank Ohl, ${ }^{4,5}$ J. Simon Wiegert, ${ }^{6}$ \\ and $\oplus_{\text {Lisa Marshall }}^{1,2}$ \\ ${ }^{1}$ Institute of Experimental and Clinical Pharmacology and Toxicology, ${ }^{2}$ Center for Brain, Behavior and Metabolism, ${ }^{3}$ Institute for Robotics and Cognitive \\ Systems, University of Luebeck, 23562 Lübeck, Germany, ${ }^{4}$ Leibniz Institute for Neurobiology, Systems Physiology of Learning, Center for Behavioral Brain \\ Sciences, 39118 Magdeburg, Germany, ${ }^{5}$ Otto-von-Guericke University Magdeburg, Faculty for Natural Sciences, 39106 Magdeburg, Germany,

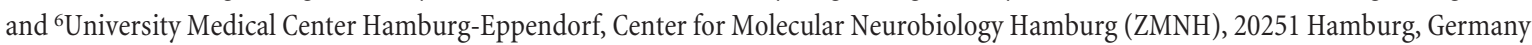

Time locking between neocortical sleep slow oscillations, thalamo-cortical spindles, and hippocampal sharp-wave ripples has convincingly been shown to be a key element of systems consolidation. Here we investigate the role of monosynaptic projections from ventral/ intermediate hippocampus to medial prefrontal cortex (mPFC) in sleep-dependent memory consolidation in male mice. Following acquisition learning in the Barnes maze, we optogenetically silenced the axonal terminals of hippocampal projections within mPFC during slow-wave sleep. This silencing during SWS selectively impaired recent but not remote memory in the absence of effects on error rate and escape latencies. Furthermore, it prevented the development of the most efficient search strategy and sleep spindle time-locking to slow oscillation. An increase in post-learning sleep sharp-wave ripple (SPWR) density and reduced time locking of learning-associated SPWR activity to sleep spindles may be a less specific response. Our results demonstrate that monosynaptic projections from hippocampus to $\mathrm{mPFC}$ contribute to sleep-dependent memory consolidation, potentially by affecting the temporal coupling of sleep-associated electrophysiological events.

Key words: Barnes maze; hippocampus; optogenetics; prefrontal cortex; sleep; spatial memory

Significance Statement

Convincing evidence supports the role of slow-wave sleep (SWS), and the relevance of close temporal coupling of neuronal activity between brain regions for systems consolidation. Less attention has been paid so far to the specific neuronal pathways underlying these processes. Here, we optogenetically silenced the direct monosynaptic projection from ventral/intermediate hippocampus (HC) to medial prefrontal cortex (mPFC) during SWS in male mice following repeated learning trials in a weakly aversive spatial task. Our results confirm the concept that the monosynaptic projection between HC and mPFC contributes to memory consolidation and support an important functional role of this pathway in shaping the temporal precision among sleep-associated electrophysiological events.

\section{Introduction}

The two-stage theory of memory formation posits that memory traces are initially encoded into hippocampus (HC), which serves

\footnotetext{
Received Aug. 22, 2018; revised June 13, 2019; accepted June 21, 2019.

Author contributions: S.B., F.O., and L.M. designed research; S.B., M.L., and S.A. performed research; S.B., M.M., and M.L. analyzed data; R.B. and J.S.W. contributed unpublished reagents/analytic tools; S.B., M.M., M.L., F.O., J.S.W., and L.M. wrote the paper.

This work was supported by DFG (SPP 1665 MA2053/4, SPP 1665 0H69/1-2, SPP 1926 WI4485/3-1, SFBTR654/ TPA6, F0R2419 WI4485/2-1) and Center for Behavioral Brain Sciences (FKZ ZS/2016/04/78120). We thank Alexey Ponomarenko (advice and discussions on optogenetics, University of Düsseldorf), Diana Campos (help with data acquisition), Lisa Kühne (scoring of sleep and behavioral video data), Katia Monsorno (scoring of behavioral video data and help with immunohistochemistry), Helge Müller-Fielitz (advice on immunohistochemistry and virus injection procedure), and Ingke Braren from the vector facility of the University Medical Center Hamburg-Eppendorf(AAV production)

The authors declare no competing financial interests.
}

as a transient storage site, and are, in the course of memory consolidation, gradually transferred to the neocortex for long-term storage. This form of systems consolidation (Marr, 1971; Frankland and Bontempi, 2005) benefits from sleep, particularly from the oscillatory rhythms occurring during deep non-rapid eye movement (NREM) sleep, the neocortical slow oscillations (SOs), thalamo-cortical spindles, and hippocampal sharp-wave ripples (SPWRs; Rasch and Born, 2013), as supported also by studies involving their modulation. SOs can induce LTP-like plasticity processes (Chauvette et al., 2012), and enhancement of

Correspondence should be addressed to Lisa Marshall at lisa.marshall@pharma.uni-luebeck.de or Sonja Binder at sonja.binder@pharma.uni-luebeck.de.

https://doi.org/10.1523/JNEUROSCI.2158-18.2019

Copyright $\odot 2019$ the authors 
neocortical SOs and spindles by exogenous stimulation has led to improved memory consolidation (Marshall et al., 2006; Ngo et al., 2013; Binder et al., 2014). Disruption of SPWRs, which are closely associated with replay of neuronal patterns, on the other hand, has substantially impaired memory consolidation and prevented memory trace stabilization (Wilson and McNaughton, 1994; Girardeau et al., 2009; Ego-Stengel and Wilson, 2010; Jadhav et al., 2012; Roux et al., 2017). Successful memory consolidation has furthermore been linked to enhanced post-learning spindle activity (Gais et al., 2002; Schabus et al., 2004; Clemens et al., 2005; Cox et al., 2012). During sleep these brain rhythms interact in a temporally precise manner (Sirota et al., 2003; Isomura et al., 2006; Mölle et al., 2006; Wierzynski et al., 2009; Peyrache et al., 2011), e.g., it has been shown that neuronal replay in medial prefrontal cortex (mPFC) occurs preferentially during SPWR episodes (Peyrache et al., 2009), and that spindles occur preferentially at the transition to or during the up-state of the SO (Mölle et al., 2006; Peyrache et al., 2009). Moreover, recent studies at the field potential and cellular level reveal a causal role of fine-tuned SPWR-spindle and spindle-delta coupling for the consolidation of memories (Maingret et al., 2016; Xia et al., 2017).

Although there is convincing evidence for the process of systems consolidation during sleep, on one hand, as well as for the close temporal relationship between neuronal activity in distributed brain regions during sleep, on the other hand, less attention has been paid so far to the specific neuronal pathways involved in these processes. Possible important routes for information transfer from hippocampus to $\mathrm{mPFC}$ include multisynaptic projections via entorhinal cortex (Swanson and Köhler, 1986; Insausti et al., 1997; Hoover and Vertes, 2007; Kitamura et al., 2017) or via thalamic nuclei (Pereira de Vasconcelos and Cassel, 2015). Furthermore, monosynaptic projections of ventral and intermediate $\mathrm{HC}(\mathrm{vHC} / \mathrm{iHC})$ to prelimbic and infralimbic (IL) regions of mPFC exist in mice and rats (Laroche et al., 2000; Thierry et al., 2000; Cenquizca and Swanson, 2007). The vHC to mPFC pathway has been studied for its role in working memory, emotional, in particular anxiety-like behavior and depression in association with theta oscillatory activity in vHC and mPFC (Adhikari et al., 2010; Spellman et al., 2015; Carreno et al., 2016; Padilla-Coreano et al., 2016), but to our knowledge the implication of this pathway for memory consolidation has not been explored.

The goal of our study was to investigate whether monosynaptic projections from $\mathrm{HC}$ to $\mathrm{mPFC}$ are critically involved in sleep-dependent memory consolidation of a spatial task. To accomplish this, we transduced the $\mathrm{vHC} / \mathrm{iHC}$ of mice with the inhibitory opsin iChloC (Wietek et al., 2015) and used optogenetic inhibition at the axonal terminals of hippocampal projections within $\mathrm{mPFC}$ during slow-wave sleep (SWS) following learning in the Barnes maze. Electrophysiological activity was assessed by local field potentials (LFPs) recorded within the dorsal HC (dHC), mPFC, and cingulate (CG) and IL. We hypothesized that the functional impairment of this pathway during SWS would result behaviorally in impaired performance on the Barnes maze task, expressed as increased errors and longer latencies to reach the target hole. Electrophysiologically we hypothesized for NREM sleep less pronounced oscillatory rhythms and/or decreased temporal coordination of SO, spindles, and SPWR.

\section{Materials and Methods}

\section{Animals}

Thirty-four male C57BL/6N mice (Janvier), 10-11 weeks old at start of the experiments, were used. Mice were housed in standard polycarbonate
IVC cages, initially in pairs and after viral injection individually on a $12 \mathrm{~h}$ light/dark cycle with ad libitum access to food and water. All animals were treated identically, i.e., received viral injections and underwent the identical implantation surgery. All mice took part in the Barnes maze experiment; however, three animals had to be excluded due to technical problems with the laser used for optogenetic inhibition and one because of misplacement of optic fibers. One additional animal had to be killed due to postsurgical complications. All procedures were performed in accordance with European and national guidelines (EU Directive 2010/ $63 / \mathrm{EU}$ ), and were approved by the local state authority (Ministerium für Energiewende, Landwirtschaft, Umwelt und ländliche Räume, Schleswig Holstein). Animals were handled for $3 \mathrm{~min} / \mathrm{d}$ for $5 \mathrm{~d}$ before the first surgery.

\section{Virus injections}

Animals were anesthetized with isoflurane (induction: $3.5 \%$, maintenance: $1.3-2.5 \%$ in $11 / \mathrm{min}_{2}$ ) and placed in a stereotactic apparatus (David Kopf Instruments). Additionally, $5 \mathrm{mg} / \mathrm{kg}$ carprofen (Rimadyl, Pfizer) was given intraperitoneally for pain relief and $0.04 \mathrm{mg} / \mathrm{kg}$ atropine (Atropinum Sulfuricum, Eifelfango) was administered subcutaneously to prevent breathing problems. Lidocaine (1\% solution, B. Braun Melsungen) was used locally before potentially painful procedures. Every $30 \mathrm{~min} 0.1 \mathrm{ml}$ of warm saline was given subcutaneously for fluid substitution. Virus injection procedures were similar as by Cetin et al. (2006). Two small craniotomies were made on each hemisphere above the $\mathrm{vHC} /$ iHC (AP: $-3.00, \mathrm{~L}: \pm 2.75$, DV: 4.56, and AP: -3.5 , L: \pm 3.37 , DV: 4.10 and 2.75). Shortly $165 \mathrm{nl}$ of virus suspension (adeno-associated virus carrying the improved chloride-conducting channelrhodopsin [iChloC] with neuron-specific promoter, plus red fluorescent protein [tdimer2], AAV9-CaMKII $\alpha$-iChloC-2A-tdimer2; Wietek et al., 2015) was administered to each of the six injection sites using a glass microcapillary (intraMark, Brand) at low speed of $\sim 75 \mathrm{nl} / \mathrm{min}$. After injection, the microcapillary was left in place for $8 \mathrm{~min}$ and then retracted slowly, with another 3 min stop halfway between injection site and brain surface. The scalp incision was sutured, $0.5 \mathrm{ml}$ of warm saline was given subcutaneously for fluid substitution, and the animal was kept under red light in its home cage until recovery from anesthesia.

\section{Electrode and optic fiber implantation}

Implantation surgery took place earliest $26 \mathrm{~d}$ after virus injection with identical anesthesia regime. For optogenetic inhibition of monosynaptic projections from $\mathrm{vHC} / \mathrm{iHC}$ to $\mathrm{mPFC}$, two optic fibers were inserted bilaterally above the IL (AP: $1.94, \mathrm{~L}: \pm 1.38$, DV: 2.87 , angle $20^{\circ}$ ). Optic fiber implants were fabricated from $100 \mu \mathrm{m}$ optic fibers and zirconia ferrules (both Thorlabs). They were combined with tungsten wires (40 $\mu \mathrm{m}$, California Fine Wire) protruding the tip of the optic fiber by $\sim 100$ $\mu \mathrm{m}$ to record LFP activity at the site of optogenetic inhibition. Two or three tungsten wires to record LFP activity were implanted into CG (AP: 1.20 , L: $0.20-0.30$, DV: $1.75-2.00)$ and an array of five tungsten wires was implanted into dorsal HC (AP: - 1.94, L: 1.50, DV: 1.55-1.65). In onehalf of the animals recording wires were placed into the right, in the others into the left hemisphere. Two stainless steel screws (Plastics One) implanted above the cerebellum (AP: $-4.80, \mathrm{~L}: 0.00)$ and the somatosensory cortex (AP: $-1.80, \mathrm{~L}: 1.80$ ), served as reference and ground electrode, respectively. Additionally, a polyimide-insulated stainless steel wire $(0.125 \mathrm{~mm}$ diameter, Plastics One) was implanted into the neck muscles to record EMG activity. Recording electrodes were connected to a plug-connector and secured to the skull with dental acrylic (SuperBond, Sun Medical; Grip Cement, 3M; and Palapress, Heraeus Kulzer). Following surgery, $0.5 \mathrm{ml}$ of warm saline was given subcutaneously for fluid substitution and $7.5 \mathrm{mg} / \mathrm{kg}$ enrofloxacin (Baytril, Bayer) was administered intraperitoneally to prevent postsurgical infections. The animal was kept under red light in its home cage until recovery from anesthesia.

\section{Electrophysiological data acquisition and SO-triggered optogenetic inhibition}

Electrodes were connected to a headstage preamplifier (HS-18MM, Neuralynx). Electrophysiological signals were amplified and sampled digitally at $4 \mathrm{kHz}$ with a low-passed filter set at $1 \mathrm{kHz}$ (Digital Lynx, 
Neuralynx). All recording sessions took place in recording boxes made of black PVC $(22 \times 22 \times 30 \mathrm{~cm}$; Fachhochschule Lübeck PROJEKT $)$ with ad libitum access to food and water. For optogenetic inhibition, the optic implants were connected by ceramic split sleeves to patch cords (both Thorlabs). These again were coupled to a split fiber (FCMM50-50A-FC, Thorlabs), which connected to a shutter (Uniblitz LS2, Vincent Associates) coupled to a $473 \mathrm{~nm}$ DPSS laser source (BL473T3, Lasercentury). The audio output of the Neuralynx system was used to feed analog signals of one recording channel to a custom stimulator (Wilde et al., 2015) capable of online detection of SO activity by individually set thresholds (see Threshold determination for optogenetic inhibition procedure during NREM sleep). Once a SO was detected, a trigger was sent to a pulse train generator (Pulse Pal, Sanworks; Sanders and Kepecs, 2014) and relayed to open the shutter for $50 \mathrm{~ms}$. Light intensity was $12-20 \mathrm{~mW}$, measured at the tip of the implanted optic fiber before implantation. The minimal delay between two consecutive pulses was set to $2 \mathrm{~s}$, as investigations on iChloC transduced hippocampal neurons indicated a tau-off value of $\sim 5 \mathrm{~s}$ (Wietek et al., 2015).

\section{Threshold determination for the optogenetic inhibition procedure during NREM sleep}

In animals receiving optogenetic inhibition during post-learning NREM sleep (OPTO), hippocampal output to mPFC was selectively inhibited during NREM sleep based on the detection of a SO-negative half wave. Thresholds for SO activity were determined off-line for each animal individually using data from the second habituation baseline recording (BSL; see Behavioral procedures). We applied a similar procedure as done by Mölle et al. (2006). First, sleep stages were assigned for $5 \mathrm{~s}$ epochs (see Data reduction and analysis). Then, the CG recording revealing the largest SO amplitude during NREM sleep was selected by visual inspection, $35 \mathrm{~Hz}$ low-pass filtered, downsampled to $250 \mathrm{~Hz}$, and then $4 \mathrm{~Hz}$ low-pass filtered. Negative half waves of SOs were detected if the duration between zero-crossings lay between 0.125 and $0.75 \mathrm{~s}$ and their amplitude exceeded a fixed threshold (mean LFP amplitude of all NREM sleep epochs ${ }^{\star} 1.25$ ). The threshold for the automatic optogenetic inhibition for all further experiments was then set at the mean +2 SD of the amplitudes of all detected negative half waves.

\section{Behavioral procedures}

Habituation to experimental conditions. After at least $7 \mathrm{~d}$ of recovery from implantation surgery, animals were brought to the experimental room where they stayed until the end of experiments. Following at least $1 \mathrm{~d}$ of adaptation to the room in the home cage, two habituation sessions to the recording procedures took place during the light phase on 2 consecutive days. After animals were placed into their recording boxes, headstage and patch cords were connected and electrophysiological activity was recorded for $3 \mathrm{~h}$. No laser pulses were given. The evening before the start of the acquisition phase of the Barnes maze task, animals were placed into the recording box without connecting them to the tethers for overnight adaptation.

Barnes maze experiment: acquisition phase. All trials in the Barnes maze (Stoelting; technical specifications: diameter $91 \mathrm{~cm}$, height $90 \mathrm{~cm}, 20$ holes, hole diameter $5 \mathrm{~cm}$ ), took place at the beginning of the light phase (lights on: 8:00 A.M.). The procedures were similar as described by Sunyer et al. (2007). The animal was placed into a lightproof start box $(11 \times$ $10 \times 6.5 \mathrm{~cm})$ in the middle of the maze and a bright light above the maze (330 lux at maze level) was turned on. After $10 \mathrm{~s}$, the start box was lifted away. Around the maze, visual cues were available for spatial orientation. On the first day only, an adaptation trial was conducted: After lifting the start box, the animal was gently guided by the experimenter to the escape box. If it did not enter the escape box on its own, it was gently placed into it. The opening to the escape box was covered with a lightproof lid and the animal remained there for $2 \mathrm{~min}$. Immediately after the adaptation trial, the acquisition trials took place. As in the adaptation trial, the animal was placed for 10 s into the start box. After lifting the start box, the animal was allowed to explore the maze for up to $3 \mathrm{~min}$. The trial ended when the animal entered the escape box with all four paws. If it did not enter after the $3 \mathrm{~min}$ had elapsed, it was gently placed into the escape box by the experimenter. The animal stayed in the escape box, covered by the lightproof lid, for $1 \mathrm{~min}$ and was then transferred to its home cage for an intertrial interval of $15 \mathrm{~min}$. The maze was cleaned with $70 \%$ ethanol solution between trials. For 4 consecutive days (Days 1-4), four acquisition trials per day were conducted. After the last trial of each day, the animal was brought into the recording box, connected to the tethers and electrophysiological activity was recorded.

Animals of the experimental group $(N=10$, OPTO) received optogenetic inhibition as described above during NREM sleep throughout the $3 \mathrm{~h}$ recording session, animals of the control group $(N=10, \mathrm{CTRL}) \mathrm{did}$ not receive any optogenetic inhibition. At the end of the recording session, the animal was disconnected from the tethering, but stayed in the recording box until the next morning. After completion of the recording session on Day 4, the animal was placed back into its home cage. All trials in the Barnes maze were digitally video recorded for off-line analysis using a camera (DMK 42AUC03, The Imaging Source) placed above the maze. Most experiments were conducted with two animals on the same day, i.e., a control animal and an experimental animal were subjected to Barnes maze acquisition and sleep recording in parallel.

Barnes maze experiment: probe trials. On Day 5 and Day 16 after the first acquisition trial, probe trials took place. The escape box was replaced by a blind. In addition, the maze was rotated by $180^{\circ}$ to prevent orientation on potentially present intra-maze cues. As before, the animal was placed into the start box, which was lifted after $10 \mathrm{~s}$. The animal was allowed to explore the maze for $90 \mathrm{~s}$, thereafter, it was brought back into its home cage.

\section{Non-learning control sessions}

To investigate effects of optogenetic inhibition on electrophysiological activity at baseline levels, i.e., without any prior learning experience, additional sleep recording sessions with and without optogenetic inhibition were conducted. Each animal was recorded on $2 \mathrm{~d}$ during the first $3 \mathrm{~h}$ of the light phase. Within one of the sessions, optogenetic inhibition procedure was applied as described above (see Data acquisition and SOtriggered optogenetic inhibition), the other session served as withinsubject control. Order of conditions was randomized and balanced. In total, 19 animals from the Barnes Maze experiment were used for this experiment (Experiment 2); including the three animals which had to be excluded from analysis of the main experiment due to malfunction of the laser.

\section{Additional control experiment: optogenetic inhibition during} wakefulness and REM sleep

To investigate the specificity of effects of the inhibition procedure for NREM sleep, an additional control group received optogenetic inhibition during post-learning periods of predominantly wakefulness and REM sleep (WRO-CTRL, $N=5$ ). Here, within a $3 \mathrm{~h}$ post-learning period, wakefulness and REM sleep were visually detected online by an experienced experimenter, who started the automatic detection of theta activity $(7.0-8.6 \mathrm{~Hz})$ in the hippocampal LFP using a fixed threshold of $130 \mu \mathrm{V}$. Detected theta activity then triggered laser pulses, as described in section 2.4 for SO-triggered optogenetic inhibition. All other procedures were identical to OPTO and CTRL.

\section{Immunohistochemistry}

At the end of experiments, animals were deeply anesthetized with Avertin ( $400 \mathrm{mg} / \mathrm{kg}$; Sigma-Aldrich) and killed by transcardial perfusion with 10 $\mathrm{ml}$ of ice-cold heparinized saline followed by $10 \mathrm{ml}$ of ice-cold $4 \%$ paraformaldehyde solution. Brains were extracted and postfixed for $\sim 15 \mathrm{~h}$ in $4 \%$ paraformaldehyde at $4^{\circ} \mathrm{C}$. To investigate virus expression and position of optic fibers, $50 \mu \mathrm{m}$ coronal vibratome slices from mPFC and vHC/iHC were prepared. Sections were rinsed $1 \times 10 \mathrm{~min}$ in tris-buffered saline (TBS) with $0.03 \%$ Triton X-100 (TBS-Tx), incubated for $1 \mathrm{~h}$ in TBS-Tx containing $5 \%$ bovine serum albumin (BSA; Sigma Aldrich) and then incubated for $\sim 15 \mathrm{~h}$ at $4^{\circ} \mathrm{C}$ in TBS-Tx containing 5\% BSA, mCherry antibody (to amplify tdimer2 fluorescence; 1:500; SICGEN, catalog \#AB0040-500; RRID:AB_2333093) and Anti-2A peptide antibody (to check for insertion of iChloC channel protein into the membrane; 1:1000; Millipore, catalog \#ABS31; RRID:AB_11214282). The sections were then rinsed $2 \times 10 \mathrm{~min}$ in TBS-Tx and incubated for $2 \mathrm{~h}$ in TBS-Tx containing 5\% BSA, AlexaFluor 488-labeled secondary anti- 
body (1:500; ThermoFisher Scientific, catalog \#A-21206; RRID: AB_2535792), Cy3-labeled secondary antibody, for 2A-tag staining (1: 1000; Jackson ImmunoResearch Laboratories, catalog \#705-165147; RRID:AB_2307351), and DAPI (1:1000; A1001, AppliChem). Following another $2 \times 10 \mathrm{~min}$ rinsing steps with TBS-Tx, slices were mounted in Mowiol 4-88/DABCO (0173, 0178; Carl Roth). Fluorescence images were taken using a $10 \times$ objective (DMI $6000 \mathrm{~B}$, Leica).

\section{Electrophysiological confirmation of optogenetic silencing}

To assess the efficiency of our axonal silencing strategy, four additional mice received viral injections into the ventral hippocampus as for the experiment proper. Thirteen weeks later, mice were anesthetized with pentobarbital $(60 \mathrm{mg} / \mathrm{kg}$, additional pentobarbital was administered to maintain areflexia), fixed in a stereotactic frame and the skull exposed. A custom-made concentric stimulation electrode was implanted into vHC (AP: $-3.7 \mathrm{~mm}$, L: $3.3 \mathrm{~mm}$, DV: $4.1 \mathrm{~mm}$ ) and a craniotomy performed above $\mathrm{mPFC}$ (coordinates as in main experiment). A custom-made optrode was subsequently lowered into the $\mathrm{mPFC}$ below the craniotomy until optimal stimulation responses were observed (105 $\mu \mathrm{m}$ core NA 0.22 multimode fiber (Thorlabs), beveled $90 \mu \mathrm{m}$ platinum/tungsten glass electrode (Thomas Recording), biosignal recording with Neuralynx DigiLynx system (sampling rate: $32 \mathrm{kS} / \mathrm{s}$; filter: $2 \mathrm{~Hz}$ to $9 \mathrm{kHz}$ ). Evoked field potentials in $\mathrm{mPFC}$ were obtained in response to single pulses in vHC (bipolar stimulation current: $800 \mu \mathrm{A}, 100 \mu$ s each phase; A-M Systems, model 2300 stimulus isolator; similar to that used by Tripathi et al., 2016). Optogenetic silencing in mPFC was performed with a $100 \mathrm{~ms}$ pulse of $20 \mathrm{~mW}$ blue light (optically shuttered, custom-made laser system based on CNI $473 \mathrm{~nm}, 100 \mathrm{~mW}$ DPSS laser). To avoid the effects of light artifacts and allow anion channel opening, onset of electrical stimulation was offset by $40 \mathrm{~ms}$ from illumination onset. As a control, every second trial, electric stimulation was given in the absence of a preceding laser pulse. Responses were filtered $(2-1000 \mathrm{~Hz})$ and averaged $>50$ repetitions (ITI $5 \mathrm{~s}$ ). To further reduce the effect of light-induced baseline shifts, the light artifact preceding electrical stimulation was fit with an exponential, extrapolated to the whole illumination duration, and subtracted from the average. Each evoked potential was normalized to a period shortly before electrical stimulation.

\section{Data reduction and analysis}

Behavioral measures: Barnes maze. Analysis of behavioral videos was conducted off-line by experienced raters blind to condition of each animal using tracking software (AnyMaze, Stoelting). Similar as described by Sunyer et al. (2007), video analyses comprised semiautomatic measurement of distance traveled and latency to reach the target hole as well as manual measurement of errors (head dips into non-target holes). As sometimes mice tend to continue exploring the maze after reaching the target hole for the first time, we only report primary measures (i.e., measures for the part of the trial before reaching the target for the first time; Harrison et al., 2006). Furthermore, a search strategy was assigned to each trial. As defined by Harrison et al. (2006), the following three search strategies were categorized: Direct search (the animal moves either directly to the target hole or to an adjacent hole before reaching the target for the first time), serial search (the animal visits at least 2 adjacent holes in a serial manner before reaching the target for the first time), or mixed search (the animal shows an unorganized search pattern). For the probe trial where no escape box was present, additionally numbers of head dips into each hole were counted to measure whether a preference for the former target location was exhibited. Probe trial performance of one CTRL animal could not be analyzed because of technical problems with the video camera.

Sleep architecture. Sleep architecture was determined from the LFP and EMG recordings using $5 \mathrm{~s}$ epochs for scoring according to standard criteria with the software SleepSign for Animals (Kissei Comtec) by an experienced rater blind to the condition of each animal. In short, "waking" (W) was identified by sustained EMG activity and mixed-frequency CG LFP, "NREM sleep" (NR) by low EMG and high-amplitude lowfrequency CG LFP with a high proportion of delta activity, "Pre-rapid eye movement sleep" (PreR) by low EMG and high-amplitude CG spindle activity, and "REM sleep" (REM) by an even lower EMG-signal and low-amplitude hippocampal LFP with high theta (5-9 Hz) activity. Sleep stages were expressed as percentage of the total $3 \mathrm{~h}$ time period.

Electrophysiology. For all NREM sleep epochs within the 3 h postlearning period analyses were conducted on LFP recordings from one CG and one IL channel of each animal. Data were analyzed using Spike2 (Cambridge Electronic Design) and custom scripts based on the built-in script language. Data of the CG LFP from one animal of CTRL had to be excluded from analyses due to a technical problem. For analysis of Experiment 2, three animals had to be excluded from analysis involving SPWR because of very low SPWR density and amplitude, probably because of electrode displacement.

To identify slow oscillations in the CG and the IL LFP signals we first applied a low-pass finite impulse response (FIR) filter of $30 \mathrm{~Hz}$ and down-sampled the resulting signal to $100 \mathrm{~Hz}$. Subsequently, a low-pass FIR filter of $3.5 \mathrm{~Hz}$ was used to produce the slow oscillation signal. In the slow oscillation signal all two succeeding positive-to-negative zero crossings separated by $0.45-1.43 \mathrm{~s}$ (corresponding to $0.7-2.22 \mathrm{~Hz}$ ) were marked and the negative and the positive peak potentials between these marked positive-to-negative zero crossings were registered. SO events were defined as those intervals that displayed (1) a negative peak amplitude of 1.25 times the average negative peak amplitude of the respective baseline session or lower (Experiment 1: second habituation session; Experiment 2: control condition of the non-learning control session), and (2) a positive-to-negative peak amplitude difference of at least 1.25 times the average positive-to-negative peak amplitude difference of the BSL session.

To identify spindles, a FIR bandpass filter of $9-15 \mathrm{~Hz}$ was applied to the low-pass filtered $(<30 \mathrm{~Hz})$ and downsampled $(100 \mathrm{~Hz})$ LFP signals of the CG and IL channels. After bandpass filtering, a root mean square (RMS) representation of the filtered signal was calculated using a sliding window of $0.2 \mathrm{~s}$ with a step size of one sample. Additional smoothing was performed with a sliding-window average of the same $0.2 \mathrm{~s}$ size. Time frames were considered as spindle intervals if the RMS signal exceeded a threshold of $1.25 \mathrm{SD}$ of the bandpass filtered signal for $0.5-3 \mathrm{~s}$ and if the largest value within the frame was $>2$ SD SD of the bandpass filtered signal. Individual thresholds were derived from the bandpass filtered signal of the baseline session for every animal. Two succeeding spindles were counted as one spindle when the interval between the end of the first spindle and the beginning of the second spindle was shorter than $0.5 \mathrm{~s}$ and the resulting (merged) spindle was not $>3 \mathrm{~s}$. Detected events were not accepted as spindles, when the difference between the largest and smallest potential of the low-pass filtered signal $(<30 \mathrm{~Hz})$ within the frame was 5 times larger than 2 SD of the bandpass filtered signal and the time between these two extrema was equal or shorter than one-half an oscillation cycle of $15 \mathrm{~Hz}(0.033 \mathrm{~s})$. To exclude false detections of spindles potentially caused by the shutter artifact, all detected spindles in an interval of $\pm 0.1 \mathrm{~s}$ around a shutter event were excluded from further analyses. For event-correlation analyses the peaks and troughs of every spindle were marked as the maxima and minima of the bandpass filtered signal (between the beginning and end of the spindle), and the deepest trough was designated as the "spindle peak" that represented the respective spindle in time, i.e., the time point taken for referencing event correlation histograms (see description of event correlation histograms later in this section).

To identify ripples in the dorsal hippocampal LFP recordings, first, we applied a low-pass FIR filter of $300 \mathrm{~Hz}$ and downsampled the resulting signal to $1000 \mathrm{~Hz}$. Subsequently, we applied a bandpass FIR filter of 150-200 Hz. After bandpass filtering, the RMS was calculated using a time window of $0.02 \mathrm{~s}$ and the resulting RMS signal was smoothed with a moving average of $0.02 \mathrm{~s}$. Time frames were considered as ripple intervals if the RMS signal exceeded a threshold of $1.25 \mathrm{SD}$ of the bandpass filtered signal for $0.025-0.1 \mathrm{~s}$ and if the largest value within the frame was $>5 \mathrm{SD}$ of the bandpass filtered signal. Individual thresholds were derived from the bandpass filtered signal of the baseline session for every animal. Detected events were not accepted as ripples, when the difference between the largest and smallest potential of the low-pass filtered signal $(<300$ $\mathrm{Hz}$ ) within the frame was 5 times larger than $5 \mathrm{SD}$ of the bandpass filtered signal and the time between these two extrema was equal or shorter than one-half an oscillation cycle of $200 \mathrm{~Hz}(2.5 \mathrm{~ms})$. For event-correlation 
A Exp. 1
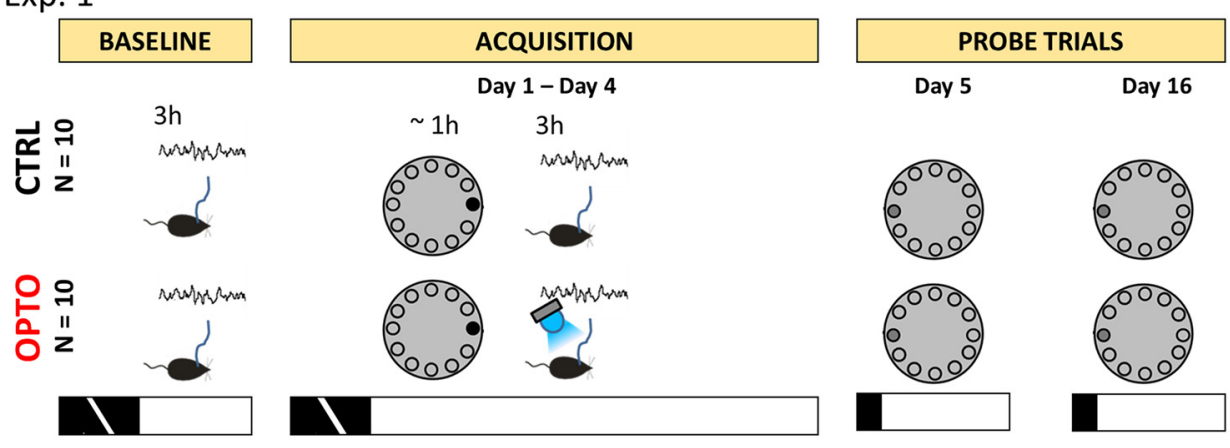

Exp. 2

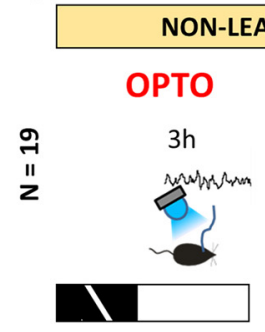

B

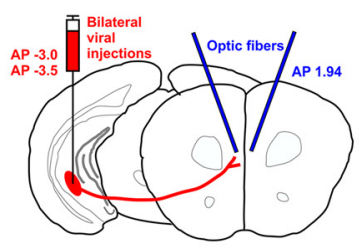

Figure 1. Sketch of experimental procedures. A, The main experiment (Experiment 1, top row) started with a baseline measurement of electrophysiological activity for $3 \mathrm{~h}$ during the early light phase. Within the following acquisition phase in the Barnes maze, animals received 4 trials/d of training, starting at the beginning of the light phase. The target escape box (black circle) did not change its position relative to available extra-maze cues throughout the acquisition phase. Immediately after Barnes maze training, electrophysiological activity was recorded again for $3 \mathrm{~h}$. Within this recording session, the OPTO received optogenetic inhibition during NREM sleep. For the $90 \mathrm{~s}$ probe trials at the beginning of the light phase on Day 5 and Day 16 , the maze was rotated by $180^{\circ}$ to prevent orientation on potentially present intra-maze cues while the extra-maze cues remained stationary. The escape box was removed and replaced by a blind (dark gray circle). In a control experiment (Experiment 2, Non-learning control), each animal was recorded on $2 \mathrm{~d}$ during the first $3 \mathrm{~h}$ of the light phase. Within one of the sessions, the optogenetic inhibition procedure was applied during NREM sleep, whereas the other session served as within-subject control. Black and white horizontal bars represent the dark and light cycle, respectively. B, Coordinates of AAV9-CaMKIII $\alpha$ iChloC-2A-tdimer2 injection sites into $\mathrm{VHC} / \mathrm{HHC}$ are schematized together with subsequent viral expression in projections of $\mathrm{HC}$ afferents to the mPFC, and the locations of the optic fiber implantations.

analyses, the peaks and troughs of every ripple were marked as the maxima and minima of the bandpass filtered signal, and the deepest trough was designated as the "ripple peak" that represented the respective ripple in time.

Event correlation histograms were calculated for spindle and ripple activity (number of peaks and troughs) with reference to the negative peak times of slow oscillations as identified in the IL LFP. Further event correlation histograms were calculated for ripple activity (number of peaks and troughs) with reference to the spindle (i.e., spindle peak, see description of spindle identifcation in the prior paragraph) in the IL LFP and for spindle activity (number of peaks and troughs) in the IL LFPs with reference to the ripples (i.e., ripple peak, see description of ripple identification in the prior paragraph). For all event correlation histograms, $2 \mathrm{~s}$ windows were used with an offset of $1.0 \mathrm{~s}$ and a bin size of 20 $\mathrm{ms}$. The histograms represent a measure for the probability of activity of one event at a given time to proceed or follow another event. The individual histograms were $z$-scored by the corresponding mean and SD of the spindle and ripple activity, respectively, for each animal during the $\pm 1 \mathrm{~s}$ interval to eliminate the considerable variability across animals and conditions. Grand mean averages of the detected SOs, spindles, and ripples and the event-correlation histograms across all animals were calculated for OPTO and CTRL.

To more specifically investigate the effect of optogenetic inhibition procedure on event correlations, in an exploratory analysis, we defined time windows within the event correlations modified by learning. It was expected that learning modulates the temporal coordination of the events-of-interest (Mölle et al., 2009), however, not necessarily within same time windows for each event correlation. Therefore, histograms of CTRL of the second habituation session (BSL) and the averaged acquisition sessions were compared bin-wise by Student's $t$ tests (two-tailed) within a range of $\pm 0.5 \mathrm{~s}$ around $t=0 \mathrm{~s}$. In each case the beginning and end of the time window were defined by the first and the last significant bins ( $\alpha$-level, $p<0.05$ ). If a single significant bin was separated by at least five nonsignificant bins from a neighboring significant bin, it was ig- nored and the start or end point of the window was set at the next significant bin, i.e., closer to $t=0 \mathrm{~s}$.

\section{Experimental design and statistical analysis}

The Barnes maze experiment was conducted in a between-subject design (CTRL vs OPTO, $N=10$ /group). The non-learning control sessions were conducted in a within-subject design $(N=19$, all animals participated in the Barnes maze experiment before).

In the Barnes maze acquisition trials, primary errors, primary latency to reach the target hole as well as primary distance traveled was subjected to ANOVAs for repeated measures. Strategy use per day was expressed as percentage of the three strategies (direct, serial, mixed) used within the four daily trials. Comparisons between conditions were conducted using Fisher's exact $\chi^{2}$ test. To analyze the change in strategy used across Days $1-4$, Friedman tests were applied. For the probe trials, head dips into non-target holes during the probe trials were averaged and the mean compared with head dips into the target hole by Student's $t$ tests (twotailed). Electrophysiological measures (sleep architecture; densities and peak-to-peak amplitude of SO, spindles, and SPWR; event-correlations) were subjected to ANOVAs for repeated measures, followed by post hoc tests where appropriate.

Data are expressed as mean \pm SEM per day if not stated otherwise and analyzed using the software package SPSS Statistics 22 software (IBM). In all ANOVAs, Huynh-Feldt corrections were applied if necessary. A $p$ value $<0.05$ was considered significant. In WRO-CTRL, only descriptive analyses were used because of low sample size $(N=5)$.

\section{Results}

\section{Barnes maze}

To investigate the contribution of the monosynaptic HC output to the $\mathrm{mPFC}$ to memory consolidation, 25 mice were trained for $4 \mathrm{~d}$ in the Barnes maze as described by Sunyer et al. (2007). As 
A

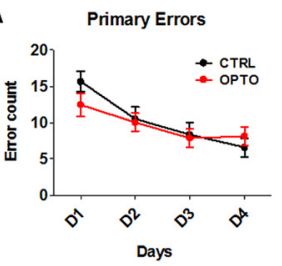

B

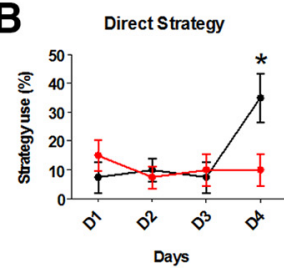

E

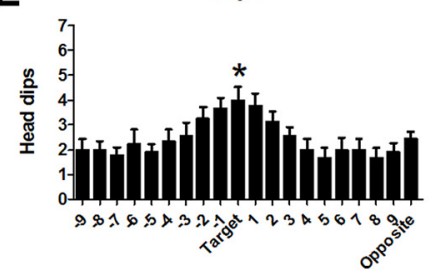

Primary Latency
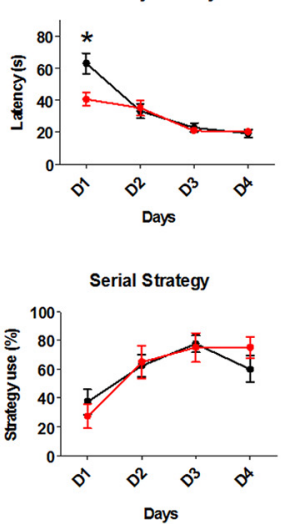

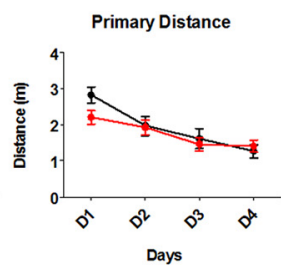

Mixed Strategy

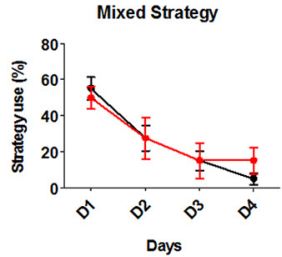

Day 5
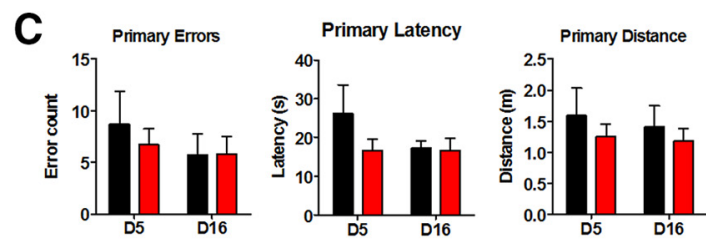

D
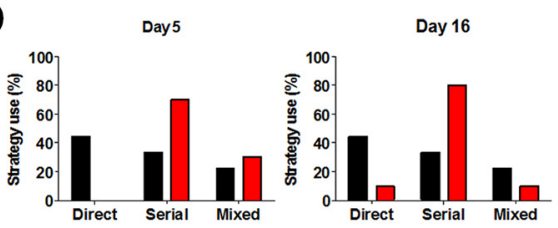
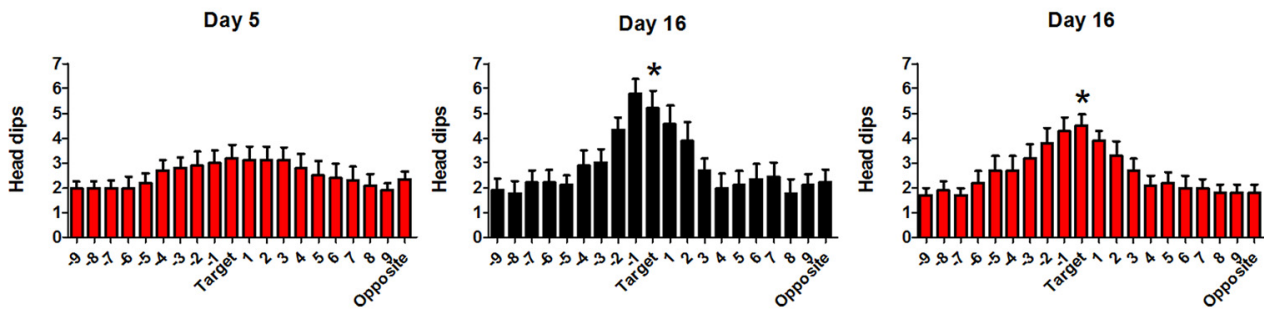

Figure 2. Barnes maze performance. $A$, Left to right, Time course across acquisition Days 1-4 (D1-D4) with four trials/d, of primary errors, primary latency and primary distance (mean \pm SEM) for CTRL and OPTO. B, Percentage of behavioral trials indicating use of direct, serial, or mixed strategy for CTRL and OPTO on acquisition Days 1-4. A significant group difference is found on Day 4, where CTRL animals increase their use of a direct strategy, whereas OPTO animals are using a direct strategy only rarely throughout all acquisition days. C, Left to right, Primary errors, primary latency, and primary distance for CTRL and OPTO on probe trial Days 5 and 16. D, Percentage of behavior indicating use of direct, serial, or mixed strategy for CTRL and OPTO on probe Days 5 and 16 . E, Number of head dips into each hole on Day 5 (left) and Day 16 (right) for CTRL and OPTO. Number of head dips into the target hole was compared against the average number of head dips across all other non-target holes (Student's $t$ tests). On Day 5, only the CTRL group showed a preference for the target location ( $p<0.05$ ), whereas the OPT0 animals explored all holes to an equal amount. On Day 16 , both groups show a significant preference for the target location (both $p<0.05$ ). Black lines, symbols, and bars: (TRL; red lines, symbols, and bars: OPT0. D1-D4: $N=10$ per group. Day 5, Day 16: CTRL $N=9,0$ PTO $N=10{ }^{*} p<0.05$. Error bars indicate SEM.

shown in Figure 1, during the acquisition phase they received four trials per day, starting at the beginning of the light phase. Immediately after completion of a training session, electrophysiological activity was recorded for $3 \mathrm{~h}$. Within this recording session, 10 mice (OPTO) received optogenetic inhibition during NREM sleep, 10 mice did not (CTRL), and an additional control group of five mice received optogenetic inhibition predominantly during wakefulness and REM sleep (WRO-CTRL). On Day 5 and Day 16, $90 \mathrm{~s}$ probe trials were conducted to test for recent and remote memory, respectively.

Figure $2 A$ reveals that performance of animals in CTRL and OPTO improved equally across the 4 acquisition days. Figure $10 \mathrm{~A}$ indicates an overall similar pattern for primary errors, latency, and distance for WRO-CTRL. Primary errors, primary latency to reach the target escape hole, as well as primary distance traveled decreased (effect of Day, primary errors: $F_{(3,54)}=10.15, p<$ 0.001 ; primary latency: $F_{(2.24,40.3)}=29.58, p<0.001$; primary distance: $F(2.8,50.6)=11.63, p<0.001)$. There were no main effects of group (primary errors: $F_{(1,18)}=0.28, p=0.604$; primary latency: $F_{(1,18)}=2.65, p=0.121$; primary distance: $F_{(1,18)}=$ $1.27, p=0.274)$. No significant interactions were found for primary errors $\left(F_{(3,54)}=1.10, p=0.368\right)$ or distance $\left(F_{(2.8,50.6)}=\right.$ $1.12, p=0.349)$. For primary latency a significant interaction was revealed $\left(F_{(2.24,40.3)}=4.44, p=0.015\right)$, which was because of a shorter latency of OPTO already on Day 1, i.e., before any manipulation took place $\left(t_{(18)}=2.84, p=0.011\right)$. Primary errors, latency, and distance are in reference to the first encounter with, i.e., head dip into, the escape hole, regardless of whether the animal entered the hole or continued further exploring the maze.
Next we analyzed changes in search strategy across the 4 acquisition days (Fig. 2B). A difference between conditions in search strategy was only found on Day $4\left(\chi_{(2)}^{2}=8.11, p=0.015\right.$; Fig. $2 B$ ), not on Days 1-3 (Day 1: $\chi_{(2)}^{2}=1.57, p=0.467$; Day 2: $\chi_{(2)}^{2}=0.24, p=1.000$; Day 3: $\left.\chi_{(2)}^{2}=0.26, p=1.000\right)$. Use of a serial strategy increased significantly (CTRL: $\chi_{(3)}^{2}=13.03, p=$ 0.002; OPTO: $\left.\chi_{(3)}^{2}=10.55, p=0.01\right)$, whereas use of a random mixed strategy significantly decreased over the acquisition phase in both groups (CTRL: $\chi_{(3)}^{2}=20.86, p<0.001$; OPTO: $\chi_{(3)}^{2}=$ $8.81, p=0.026)$. A direct strategy was used in OPTO animals only rarely, a pattern which remained stable across the acquisition phase $\left(\chi_{(3)}^{2}=0.96, p=0.835\right)$, whereas CTRL revealed a significant change in direct strategy over time $\left(\chi_{(3)}^{2}=9.40, p=0.019\right)$, an effect apparently due to the strong increase in using the direct strategy on Day 4. Indeed, group comparisons of strategy used on Day 4 revealed a significant difference for the direct, but not for the other two strategies (direct: $U=-2.26, p=0.031$; mixed: $U=-0.80, p=0.373$; serial: $U=-1.18, p=0.305$ ). Figure $10 B$ indicates that, similar to CTRL, WRO-CTRL obtained a direct strategy on Day 4 and continued using it on Day 5 (compare Figs. $2 D, 10 C)$.

On probe trials Days 5 and 16, significant group differences did not occur for either primary errors, latency or distance (Day 5: errors: $t_{(17)}=-0.56, p=0.586$; latency: $t_{(17)}=-1.23, p=0.246$; distance: $t_{(17)}=-0.69, p=0.503$; Day 16: errors: $t_{(17)}=-0.05, p=$ 0.960 ; latency: $t_{(17)}=0.19, p=0.853$; distance: $t_{(17)}=0.60, p=$ 0.557; Fig. $2 C$ ). Comparisons between the groups on the strategy used did reveal a trend for the probe trial of Day $5\left(\chi_{(2)}^{2}=5.44\right.$, $p=0.087$ ), but no significant difference for the trial on Day 16 
Table 1. F statistics for sleep architecture of the acquisition period

\begin{tabular}{|c|c|c|c|c|c|c|c|c|}
\hline & \multicolumn{2}{|c|}{ WAKE } & \multicolumn{2}{|c|}{ NREM } & \multicolumn{2}{|l|}{ REM } & \multicolumn{2}{|c|}{ PreREM } \\
\hline & $F$ & $p$ & $F$ & $p$ & $F$ & $p$ & $F$ & $p$ \\
\hline Group & 0.78 & 0.781 & 0.87 & 0.873 & 1.21 & 0.286 & 2.98 & 0.102 \\
\hline Time & 0.93 & 0.435 & 1.38 & 0.260 & 2.90 & $0.043^{*}$ & 0.29 & 0.704 \\
\hline Interaction & 1.43 & 0.245 & 1.04 & 0.384 & 1.83 & 0.153 & 1.06 & 0.346 \\
\hline
\end{tabular}

Degrees of freedom, group: $F_{(1,18)}$; time and interaction: $F_{(3,54)}$; Huynh-Feldt corrections were used if necessary. ${ }^{*} p \leq 0.05$, ANOVAs for repeated measures.

$\left(\chi_{(2)}^{2}=4.18, p=0.139\right)$. It is to note, however, that none of OPTO animals applied a direct search strategy on Day 5 (Fig. 2D). On the probe trial of Day 5, only CTRL showed a significant preference for the former target location as indicated by head dips $\left(t_{(8)}=-3.43, p=0.009\right.$; Fig. $\left.2 E\right)$, whereas OPTO failed to show a preference $\left(t_{(9)}=-1.72, p=0.119\right)$. On Day 16 , however, both groups showed a significant preference for the former target location (CTRL: $t_{(8)}=-3.38, p=0.01$; OPTO: $t_{(9)}=-5.27, p=$ 0.001). As depicted in Figure 10D, in WRO-CTRL the pattern of head dips for recent memory testing on Day 5 is more pronounced than for OPTO, although less pronounced than for CTRL.

\section{Sleep architecture}

Sleep architecture was not affected by the optogenetic inhibition procedure. During the $3 \mathrm{~h}$ post-learning sleep period, the amount of time spent in the three sleep states and wakefulness did not differ between groups (Table 1; Fig. 3). Across the $4 \mathrm{~d}$ of acquisition in both groups, the amount of REM sleep increased significantly, which may reflect a more consolidated sleep. The amount of wakefulness, NREM, and preREM sleep remained stable across the days of acquisition.

\section{Density and amplitude of neocortical SOs, sleep spindles, and hippocampal SPWRs}

Learning typically modulates key features of post-learning sleep. Specifically, density and/or amplitude of the SOs, sleep spindles, and hippocampal SPWRs can be enhanced by learning compared with baseline sleep, as well as their temporal coupling (Eschenko et al., 2006; Mölle et al., 2009; Maingret et al., 2016). One question was therefore whether acquisition training in the Barnes maze would modulate these features of SWS, and second, whether features of these rhythms would be affected by our optogenetic inhibition procedure. For this purpose we compared densities and peak-to-peak amplitudes of SOs, sleep spindles, and SPWRs between sleep during the BSL recording and after the first day of acquisition training (Day 1). Densities and peak-to-peak amplitudes during baseline NREM sleep are given in Table 2 .

As depicted in Figure 4, acquisition training on Day 1 enhanced SO density and amplitude in the CG equally in both groups $(p<0.01$; Table 3$)$. In the IL SO parameters remained, however, unchanged. Similarly, density of sleep spindles was increased by learning on Day 1 in both groups in the CG $(p<0.05)$, but only tended to be enhanced in the IL $(p=0.068)$. An increase in spindle amplitude, in contrast, was only seen in the IL, but failed to reach significance in the CG (IL: $p<0.05$, CG: $p=$ 0.099). Detailed $F$ statistics on SO and spindle parameters are given in Table 3. SPWR density on Day 1 was enhanced by acquisition training in both groups $(p<0.001)$, an effect which tended to be more pronounced in OPTO $(p=0.064)$. SPWR amplitude tended to be enhanced by learning in OPTO only ( $p=0.052$ ). Detailed $F$ statistics on SPWR parameters for acquisition training on Day 1 are given in Table 4 (left).
To investigate at the electrophysiological level, whether the monosynaptic $\mathrm{HC}$ output to the $\mathrm{MPFC}$ contributes significantly to information transfer and coupling of hippocampal and neocortical rhythms during NREM sleep we tested for group differences across the acquisition phase (Days 1-4). Because of interindividual variability in parameters of the events-of-interest, results are given as baseline corrected values. As depicted in Figure 4, $A$ and $B$, neither SO density nor amplitude differed across the 4 day acquisition phase between groups, neither in the CG nor in the IL (Table 5). Similarly, no significant modulations of spindle density in either CG or IL nor of spindle amplitude in CG were found (Fig. 4C,D; Table 5). Although analysis of spindle amplitude in IL revealed a significant interaction $(p<0.05$; Fig. $4 D$; Table 5), post hoc tests failed to detect a differential modulation of the groups on any of the acquisition days (all $p$ values $>0.164$ ). Detailed $F$ statistics on SO and spindle parameters are given in Table 5. SPWR density was in both groups highest on the first day of the acquisition phase and decreased over time $(p<0.001$; Fig. $4 E$; Table 4). Animals of OPTO showed higher SPWR density across all days $(p<0.05)$, and a differential modulation within the $4 \mathrm{~d}$ of the groups was indicated by a significant interaction $(p<0.01$; Table 4$)$. Post hoc tests confirmed significantly higher SPWR densities in OPTO on Days 1 and $2(p<0.05$; Fig. $4 E)$. As depicted in Figure 10E, SPWR density of WRO-CTRL on Days 1 and 2 similarly showed a strong enhancement. SPWR amplitude was not differentially modulated in CTRL and OPTO (Fig. 4E; Table 4). Detailed $F$ statistics on modulation of SPWR parameters across acquisition days are given in Table 4 (right).

To control for any possible effects of the optogenetic inhibition on brain electrophysiological activity independent of learning Experiment 2 was conducted in which, using a within-subject design, activity at a comparable circadian time without previous learning was recorded $(N=19)$. In one of the two sessions, optogenetic inhibition during NREM sleep was applied.

Results of the non-learning Experiment 2 are depicted in Figure 5. Neither density nor amplitude of SOs (Fig. 5A) differed between conditions in either the CG (density: $t_{(18)}=1.70, p=$ 0.107 ; amplitude: $t_{(18)}=0.29, p=0.778$ ) or IL (density: $t_{(18)}=$ 1.03, $p=0.319$; amplitude: $\left.t_{(18)}=0.65, p=0.527\right)$. Similarly, spindle amplitude did not differ between conditions in either of the investigated cortical regions (CG: $t_{(18)}=-1.67, p=0.111$; IL: $\left.t_{(18)}=-1.02, p=0.322\right)$. Although spindle density in the CG remained unaltered $\left(t_{(18)}=-1.34, p=0.197\right)$, in the IL the optogenetic inhibition procedure significantly reduced spindle density $\left(t_{(18)}=2.84, p=0.011\right)$, an effect not observed in the main experiment. Most importantly, in contrast to the main experiment, optogenetic inhibition alone did not significantly affect SPWR density or amplitude (density: $t_{(15)}=-1.71, p=$ 0.108 ; amplitude: $\left.t_{(15)}=-1.77, p=0.097\right)$.

\section{Temporal coupling of neocortical SOs, sleep spindles, and hippocampal SPWRs}

To test our hypothesis, that optogenetic inhibition of the axonal terminals of $\mathrm{vHC} / \mathrm{iHC}$ neurons in the mPFC during NREM sleep would disturb the close temporal coordination of hippocampal and neocortical rhythms, we investigated activity of these rhythms time-locked to SO, spindle, and SPWR events.

Event correlation histograms were calculated for spindle and ripple activity (number of peaks and troughs) with reference to the negative peak of the slow oscillation as identified in the IL LFP. Event correlation histograms were furthermore calculated for ripple activity (number of peaks and troughs) with reference to the maximal spindle trough in the IL LFP and for spindle 

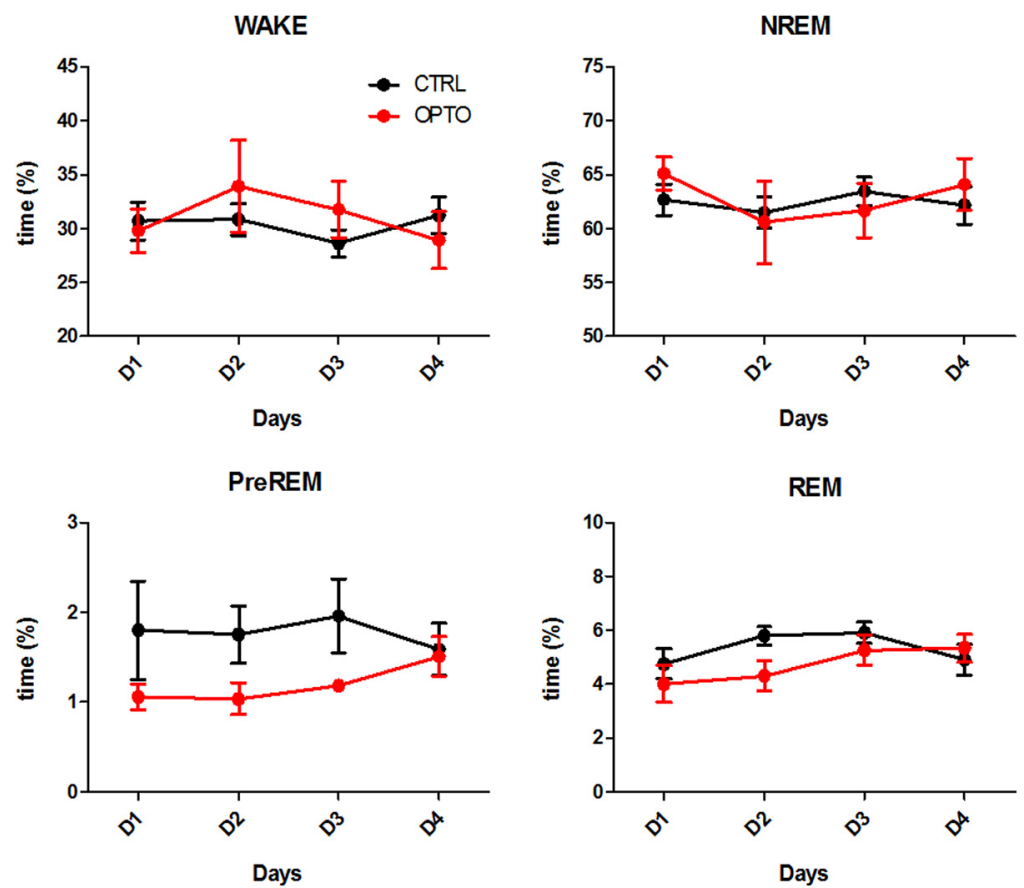

Figure 3. Sleep architecture. Mean time spent in each sleep stage expressed as percentage of the total $3 \mathrm{~h}$ recording period following each of the four acquisition trials on Days $1-4 . N=10$ per group. Error bars indicate SEM.

Table 2. Density and peak-to-peak amplitude of cortical SOs, spindles, and hippocampal SPWRs during baseline NREM sleep

\begin{tabular}{lcll}
\hline & Density, min $^{-1}$ & $\begin{array}{l}\text { Peak-to-peak } \\
\text { amplitude, } \mu \mathrm{V}\end{array}$ & $N$ \\
\hline SOs & & & \\
$\quad$ Cingulate cortex & $11.69 \pm 0.49$ & $615.40 \pm 38.69$ & 19 \\
$\quad \begin{array}{l}\text { Infralimbic cortex } \\
\text { Spindles }\end{array}$ & $9.32 \pm 0.48$ & $548.70 \pm 27.69$ & 20 \\
$\quad$ Cingulate cortex & $2.06 \pm 0.14$ & $398.61 \pm 15.20$ & 19 \\
$\quad$ Infralimbic cortex & $1.86 \pm 0.08$ & $332.47 \pm 5.99$ & 20 \\
SPWRs & $6.61 \pm 0.66$ & $297.29 \pm 21.42$ & 20 \\
\hline
\end{tabular}

Values represent mean \pm SEM

activity (number of peaks and troughs) in the IL LFPs with reference to the maximal ripple trough. Statistical analyses of all eventcorrelation histograms were conducted for time windows sensitive to learning-induced modulation (see Materials and Methods).

The event-correlation histograms of Figure $6 \mathrm{~A}$ show the typical mean decrease in spindle activity at the negative peak of the $\mathrm{SO}$, and a mean increase 100-400 ms later in line with the timing of the SO up-state. dHC SPWRs follow this temporal pattern of coupling to the SO (Fig. $6 B$ ). Figure 6, $C$ and $D$, reveal the typical nesting of SPWRs in spindle troughs and the occurrence of spindle activity during SPWRs, respectively. Learning increased the modulation of IL spindle as well as dHC SPWR activity by IL SO during the specified time window in all groups [effect of session type (BSL vs acquisition), spindles: $F_{(1,18)}=14.73, p=$ 0.001; SPWR: $F_{(1,18)}=22.14, p<0.001$; Figs. $\left.6 A, B, 10 F\right]$. Similarly, SPWR activity time-locked to IL spindles was modulated by learning within the acquisition phase more strongly than during BSL $\left(F_{(1,18)}=65.87, p<0.001\right.$; Figs. $\left.6 C, 10 F\right)$. Learning also led to a stronger modulation of spindles timelocked to SPWRs in the given time window $\left(F_{(1,18)}=76.34\right.$, $p<0.001$; Figs. $6 D, 10 F)$.
A significant effect of group $\left(F_{(1,18)}=\right.$ 4.71, $p=0.044$; Fig. $6 A$ ) was found for modulation of IL spindle activity by IL SOs. A strong trend toward an interaction $\left(F_{(1,18)}=4.09, p=0.058\right)$ indicated a differential modulation by learning. Post hoc tests indicated a significant difference between groups within the given window for the acquisition phase $\left(t_{(18)}=-2.48, p=\right.$ $0.023)$, but not for BSL $\left(t_{(18)}=-1.14, p=\right.$ $0.269)$. In WRO-CTRL, the time course of learning-induced modulation in IL spindle activity by $\mathrm{SO}$ resembled changes in CTRL (Fig. 10F). Modulation of SPWR activity by SO did not show an overall group effect for OPTO and CTRL $\left(F_{(1,18)}\right.$ $=0.45, p=0.507$; Fig. $6 B)$, however a significant interaction indicated differential modulation between these groups from BSL to acquisition $\left(F_{(1,18)}=7.66\right.$, $p=0.013)$. Post hoc-tests indicated only a trend toward a difference between groups within the acquisition phase $\left(t_{(18)}=\right.$ $-1.86, p=0.079)$, and again no difference in modulation during BSL $\left(t_{(18)}=\right.$ $-1.46, p=0.162)$. As depicted in Figure $6 C$, modulation of SPWR activity by spindles showed a significant effect of group $\left(F_{(1,18)}=6.81, p=\right.$ $0.018)$ together with a trend toward interaction $\left(F_{(1,18)}=3.52\right.$, $p=0.077)$. Post hoc tests indicated a significant difference between groups within the acquisition phase $\left(t_{(18)}=3.18, p=\right.$ $0.009)$, but a comparable modulation during $\mathrm{BSL}\left(t_{(18)}=1.15\right.$, $p=0.265$ ). Here, the time course of learning-induced modulation of SPWR activity by spindles in WRO-CTRL resembled the modifications in OPTO (Fig. 10F). The learning-dependent increase in spindle activity time-locked to SPWRs was not affected by the group (effect of group: $F_{(1,18)}=0.37, p=0.550$; interaction: $F_{(1,18)}=2.49, p=0.132$; Fig. $6 D$ ).

Together, the optogenetic inhibition procedure during postlearning NREM sleep apparently reduced both the learningdependent modulation of spindle activity time-locked to SOs as well as the modulation of SPWR activity time-locked to spindles.

With the exception of SPWR activity time-locked to the SO, temporal coordination of events was very weak under baseline conditions. Whereas in Experiment 1 the effect of optogenetic inhibition was measured between different groups of animals, in Experiment 2 the effect of the optogenetic inhibition procedure in a within-subject design without preceding learning was investigated (Figs. 1, 7). Neither spindle nor SPWR activity were differentially modulated between conditions by IL SO (SO-spindles: $t_{(18)}=-1.19, p=0.249$; Fig. $7 A$; SO-SPWR: $t_{(15)}=-1.17, p=0.261$; Fig. $\left.7 B\right)$. Yet similar to Experiment 1, modulation of SPWR activity by IL spindles was reduced in the OPTO condition $\left(t_{(15)}=3.15, p=0.007\right.$; Fig. $\left.7 C\right)$. A slight increase in modulation of spindle activity by SPWRs failed to reach significance $\left(t_{(15)}=1.99, p=0.064\right.$; Fig. $\left.7 D\right)$.

\section{Distribution of laser pulses}

Figure $8 \mathrm{~A}$ shows the distribution of laser pulse triggers and their corresponding density within each sleep state and wakefulness across the $3 \mathrm{~h}$ recording period in Experiment 1 . The majority of laser pulses (for positioning of optic fibers see Fig. 9B) were delivered as intended during NREM sleep $(86.9 \pm 2.1 \%)$, at a density of $14.7 \pm 0.6 \mathrm{~min}^{-1}$, i.e., on average every $4.1 \mathrm{~s}$ (Fig. $8 B$ ). 
A

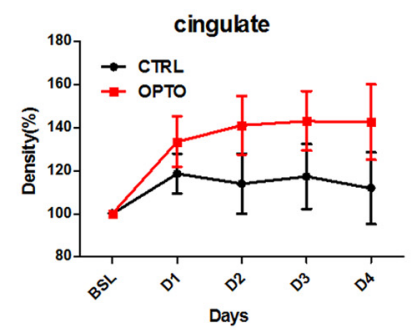

So Density

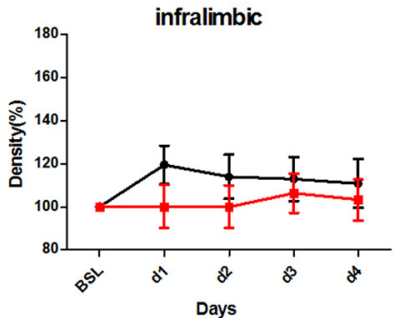

C

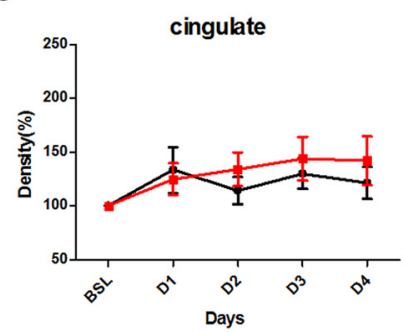

E
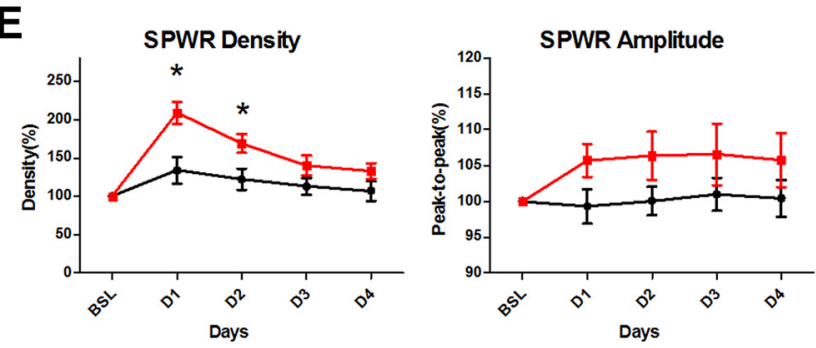

B

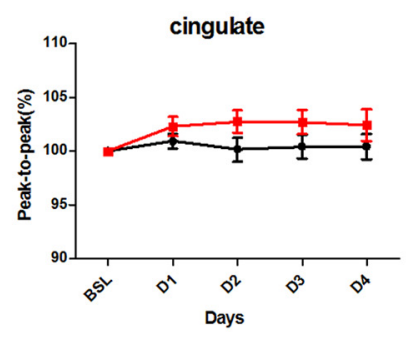

So Amplitude

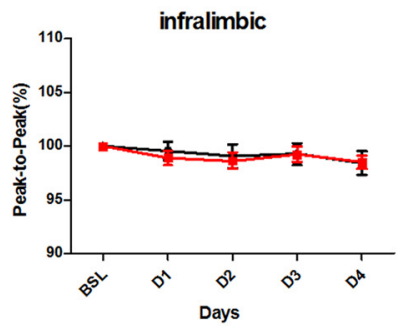

D Spindle Amplitude

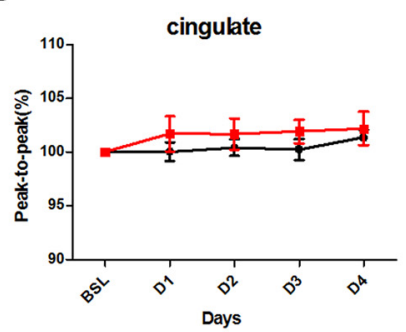

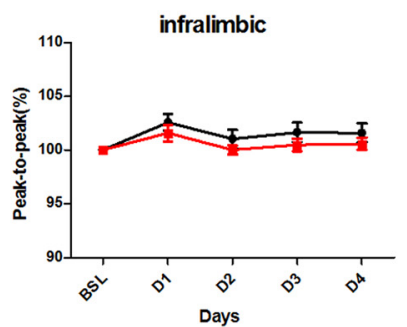

Figure 4. SOs, spindles, and SPWRs during post-learning NREM sleep of acquisition Days 1-4 compared with BSL. A, Percentage increase in SO density in CG (left) and IL (right). B, Peak-to-peak amplitude of SOs in CG (left) and IL (right). C, Percentage increase in spindle density in CG (left) and IL (right). D, Peak-to-peak amplitude of spindles in CG (left) and IL (right). $\boldsymbol{E}$, Percentage increase in SPWR density (left), and peak-to-peak SPWR amplitude (right). Learning modulates SPWR density in the OPTO significantly stronger compared with the CTRL during the first 2 acquisition days. For CG: CTRL $N=9, O P T O N=10$; for IL and hippocampus: $N=10$ per group. Error bars indicate SEM. ${ }^{*} p<0.05$.

Table 3. $F$ statistics for $S 0$ and sleep spindles: effect of learning (BSL vs Day 1)

\begin{tabular}{|c|c|c|c|c|c|c|c|c|}
\hline & \multicolumn{2}{|c|}{ SO density } & \multicolumn{2}{|c|}{$\begin{array}{l}\text { SO peak-to-peak } \\
\text { amplitude }\end{array}$} & \multicolumn{2}{|c|}{ Spindle density } & \multicolumn{2}{|c|}{$\begin{array}{l}\text { Spindle peak-to-peak } \\
\text { amplitude }\end{array}$} \\
\hline & $F$ & $p$ & $F$ & $p$ & $F$ & $p$ & $F$ & $p$ \\
\hline \multicolumn{9}{|c|}{ Cingulate cortex } \\
\hline Group & 0.11 & 0.740 & 1.77 & 0.202 & 0.04 & 0.841 & 3.04 & 0.099 \\
\hline Time & 14.05 & $<0.010^{* *}$ & 9.90 & $<0.010^{* *}$ & 6.06 & $<0.050^{*}$ & 0.70 & 0.414 \\
\hline Interaction & 0.36 & 0.558 & 1.12 & 0.306 & 0.03 & 0.861 & 0.93 & 0.350 \\
\hline \multicolumn{9}{|c|}{ Infralimbic cortex } \\
\hline Group & 1.17 & 0.294 & 1.24 & 0.280 & 0.14 & 0.717 & 0.08 & 0.784 \\
\hline Time & 1.02 & 0.326 & 2.78 & 0.113 & 3.76 & 0.068 & 5.05 & $<0.050^{*}$ \\
\hline Interaction & 2.28 & 0.148 & 0.28 & 0.605 & 0.08 & 0.786 & 0.70 & 0.415 \\
\hline
\end{tabular}

Although the density of pulses during preREM sleep reached $\sim$ half that of NREM sleep, the percentage of laser pulses during preREM sleep was $<1 \%$. Furthermore, preREM sleep only constituted $\sim 1-2 \%$ of the whole recording session. Figure $10 G$ shows the distribution of laser pulses for WRO-CTRL. As intended, the majority of pulses were given during wakefulness and REM $(83.3 \pm 2.0 \%)$; however, off-line analyses showed that $16.3 \pm 2.4 \%$ of pulses were also delivered during NREM sleep. Because of the higher percentage of NREM sleep ( 65\%) during the first $3 \mathrm{~h}$ of the post-learning sleep period the total number of laser pulses delivered in OPTO was larger than for WRO-CTRL $(1994.2 \pm 69.6$ and $838.5 \pm 71.4$, respectively).
Table 4. F statistics for SPWR

\begin{tabular}{|c|c|c|c|c|c|c|c|c|}
\hline & \multicolumn{4}{|c|}{ Effect of learning (BSL vs Day 1) } & \multicolumn{4}{|c|}{ Acquisition phase (Days 1-4) } \\
\hline & \multicolumn{2}{|c|}{ SPWR density } & \multicolumn{2}{|c|}{$\begin{array}{l}\text { SPWR peak-to-peak } \\
\text { amplitude }\end{array}$} & \multicolumn{2}{|c|}{ SPWR density } & \multicolumn{2}{|c|}{$\begin{array}{l}\text { SPWR peak-to-peak } \\
\text { amplitude }\end{array}$} \\
\hline & $F$ & $p$ & $F$ & $p$ & $F$ & $p$ & $F$ & $p$ \\
\hline Group & 2.88 & 0.107 & 2.36 & 0.142 & 6.80 & $<0.050^{*}$ & 2.23 & 0.153 \\
\hline Time & 22.93 & $<0.001^{* *}$ & 1.00 & 0.330 & 20.80 & $<0.001^{* *}$ & 0.44 & 0.658 \\
\hline Interaction & 3.91 & 0.064 & 4.34 & 0.052 & 5.13 & $<0.010^{* *}$ & 0.12 & 0.897 \\
\hline
\end{tabular}

Degrees of freedom, effect of learning: $F_{(1,18)}$; acquisition phase, group: $F_{(1,18)}$, time and interaction: $F_{(3,54)}$; Huynh-Feldt corrections were used if necessary. ${ }^{*} p \leq 0.05,{ }^{* *} p \leq 0.01$, ANOVAs for repeated measures.

Table 5. $F$ statistics for $\mathrm{SO}$ and sleep spindles throughout the acquisition phase (Days 1-4)

\begin{tabular}{|c|c|c|c|c|c|c|c|c|}
\hline & \multicolumn{2}{|c|}{ SO density } & \multicolumn{2}{|c|}{$\begin{array}{l}\text { S0 peak-to-peak } \\
\text { amplitude }\end{array}$} & \multicolumn{2}{|c|}{ Spindle density } & \multicolumn{2}{|c|}{$\begin{array}{l}\text { Spindle peak-to-peak } \\
\text { amplitude }\end{array}$} \\
\hline & $F$ & $p$ & $F$ & $p$ & $F$ & $p$ & $F$ & $p$ \\
\hline \multicolumn{9}{|c|}{ Cingulate cortex } \\
\hline Group & 1.70 & 0.210 & 1.99 & 0.177 & 0.25 & 0.621 & 0.68 & 0.422 \\
\hline Time & 0.20 & 0.795 & 0.09 & 0.897 & 0.75 & 0.530 & 2.03 & 0.122 \\
\hline Interaction & 0.78 & 0.454 & 0.69 & 0.501 & 1.21 & 0.315 & 0.51 & 0.676 \\
\hline \multicolumn{9}{|c|}{ Infralimbic cortex } \\
\hline Group & 0.78 & 0.388 & 0.05 & 0.826 & 0.77 & 0.392 & 0.46 & 0.505 \\
\hline Time & 0.41 & 0.731 & 1.98 & 0.127 & 0.30 & 0.750 & 1.89 & 0.143 \\
\hline Interaction & 1.60 & 0.204 & 0.41 & 0.747 & 1.74 & 0.188 & 3.24 & $<0.05^{*}$ \\
\hline
\end{tabular}

Degrees of freedom for $C G$, group: $F_{(1,17)}$, time and interaction: $F_{(3,51)}$; degrees of freedom for $\mathrm{L}$, group: $F_{(1,18)}$, time and interaction: $F_{(3,54)}$; Huynh-Feldt corrections were used if necessary. ${ }^{*} p \leq 0.05$, ANOVAs for repeated measures. 

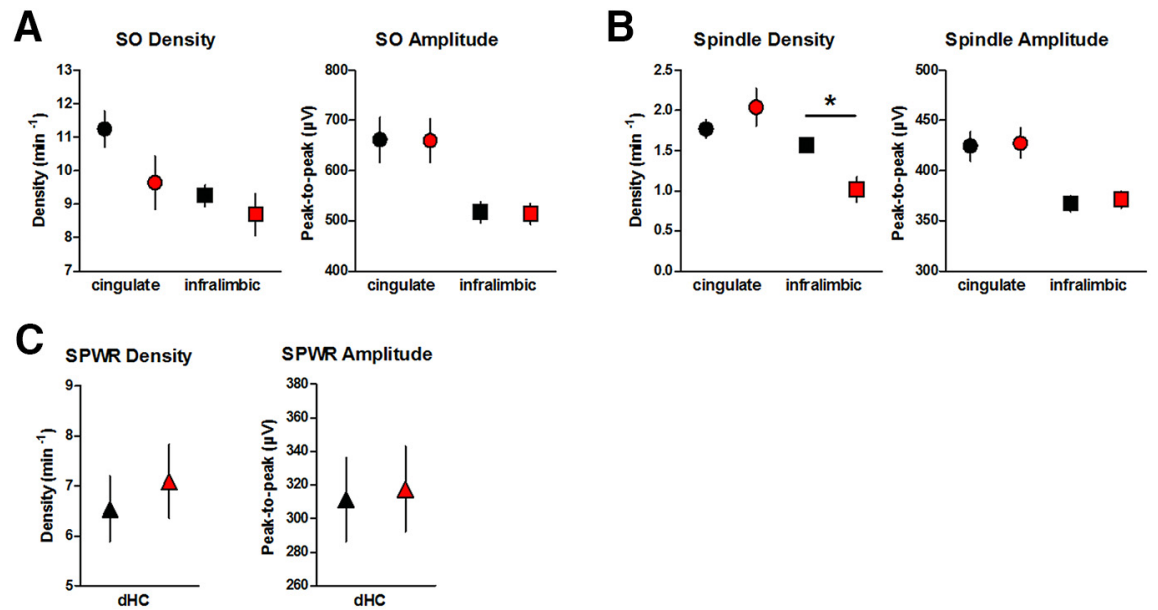

Figure 5. SOs, spindles, and SPWRs during NREM sleep in the non-learning control Experiment 2. $\boldsymbol{A}$, Mean density per minute (left) and amplitude (right) of $\mathrm{SO}$ in CG (circles) and IL (squares) $\boldsymbol{B}$, Mean density per minute (left) and amplitude (right) of spindles in CG (circles) and IL (squares). Spindle density in IL is significantly reduced in the OPTO condition. $C$, Mean density per minute (left) and peak-to-peak amplitude (right) of SPWR in dHC. For CG and IL: $N=19$; for hippocampus: $N=16$. Within-subject design. Red symbols, OPT0; black symbols: CTRL. Error bars indicate SEM. ${ }^{*} p<0.05$.

\section{Virus expression and fiber placement}

Virus expression and optic fiber placement were thoroughly checked in all animals. One animal was excluded because of fiber misplacement. Figure $9 A$ reveals a representative example of virus expression and the locations of optic fiber tips in the PRL/IL regions. The $\mathrm{vHC}$-evoked field potential in $\mathrm{mPFC}$ following application of the laser pulse was measured in anesthetized animals. The reduction in amplitude of the evoked field potential on illumination is shown in a representative animal representatively in Figure 9C.

\section{Discussion}

This study aimed to delineate the role of the monosynaptic vHC/ iHC-mPFC pathway in sleep-associated consolidation of spatial memory by applying an optogenetic silencing strategy to the hippocampal fibers within the mPFC during post-learning NREM sleep. In summary, at the behavioral level, learning rate within the $4 \mathrm{~d}$ acquisition phase was not affected by the optogenetic inhibition procedure, in contrast to our hypothesis (Fig. 2). On the other hand, OPTO was not as efficient in search strategy as CTRL on acquisition Day 4. Animals subjected to our silencing procedure applied predominantly during wakefulness and REM sleep behaved like CTRL. Recent memory on Day 5 was impaired in OPTO: animals failed to locate the target hole. Interestingly, remote memory on Day 16 was not significantly impaired in OPTO.

At the electrophysiological level, all groups showed a postlearning increase in SPWR density as reported previously (Eschenko et al., 2008; Ramadan et al., 2009). Surprisingly, this increase in post-learning SPWR density within the first days of acquisition was higher for OPTO than CTRL. Furthermore, in line with the general hypothesis on temporal coupling, modulation of SPWR activity by sleep spindles and of sleep spindle activity by $\mathrm{SO}$ was reduced in OPTO. In WRO-CTRL modulation of sleep spindle activity by SO resembled that in CTRL, however, mean SPWR density was even more enhanced than in OPTO. Also, the time course of modulation in SPWR activity by sleep spindles in WRO-CTRL appeared similar to that in OPTO.

Our optogenetic silencing approach during NREM sleep apparently did not impair acquisition of the task per se (i.e., "there is an escape box which needs to be found") as seen by the simi- larity to CTRL in basic learning measures such as error rate, latency and distance traveled. However, the failure to adopt a spatial ("direct") search strategy over time, together with the pronounced impairment in the recent memory probe trial on Day 5, indicate a lack of accuracy in spatial memory of OPTO, i.e., to remember the precise location of the escape box. Memory traces encoded into hippocampal and neocortical networks presumably undergo systems consolidation for long-term stabilization (Bontempi et al., 1999; Maviel et al., 2004; Kitamura et al., 2017). Within this gradual process, sleep associated fine-tuned communication between hippocampus and neocortex is essential (Maingret et al., 2016; Latchoumane et al., 2017). We assume that silencing $\mathrm{vHC} / \mathrm{iHC}$ output to mPFC during SWS distorted this communication and thereby the initiation of memory trace stabilization, resulting in impaired recent memory performance. Interestingly, remote memory performance was intact, which at first glance appears to be contradictory. However, remote memory consolidation is a process that takes place over days to weeks (Bontempi et al., 1999; Maviel et al., 2004; Kitamura et al., 2017). Because axonal silencing of vHC/ iHC-mPFC projections did not occur between Day 5 and Day 16, i.e., between recent and the remote memory probe trials, systems consolidation of any residual memory trace could have taken place undisturbed during this time.

Our silencing procedure during the selected brain states was not complete. First, it was restricted to states within the first $3 \mathrm{~h}$ of post-learning sleep, where reactivation is typically strongest, and here in OPTO only time periods of SWS associated with detected SOs were targeted. As in OPTO our intention was the investigation of SWS-associated memory consolidation, communication between $\mathrm{vHC}$ and $\mathrm{mPFC}$ was left essentially undisturbed in wakefulness and REM sleep. In WRO-CTRL, in which silencing targeted wakefulness and REM sleep, inhibition may have also affected replay, as SPWR during wakefulness states can be relevant for learning and memory (Foster and Wilson, 2006; Diba and Buzsáki, 2007; Roux et al., 2017). Furthermore, to prevent thermal effects on brain tissue and light-induced artifacts in recordings, illumination to shut down neuronal activity at the axonal terminals was not provided constantly, but on average every $4 \mathrm{~s}$. iChloC is an opsin with slow off-kinetics that has been shown to silence neuronal activity for 3-5 s (Wietek et al., 2015). Second, although our optogenetic approach decreased the evoked afferent activity, it does not completely suppress it. Thus, our results show the vHC/iHC-mPFC pathway during NREM sleep in the mouse is functionally relevant at least for recent SWS-dependent consolidation of certain aspects of spatial memories.

The impairment in accuracy of memory by silencing the $\mathrm{vHC/}$ $\mathrm{iHC}-\mathrm{mPFC}$ pathway is also interesting from a more theoretical perspective. Interest in the nature of information conveyed between the hippocampus and PFC, as dependent upon brain state and specific pathways involved, is increasing (Spellman et al., 2015; Eichenbaum, 2017; Tang and Jadhav, 2019). Our results on the failure to develop the correct strategy despite normal learning rate may indicate a certain dependence on the $\mathrm{vHC} / \mathrm{iHC}-\mathrm{mPFC}$ 
A
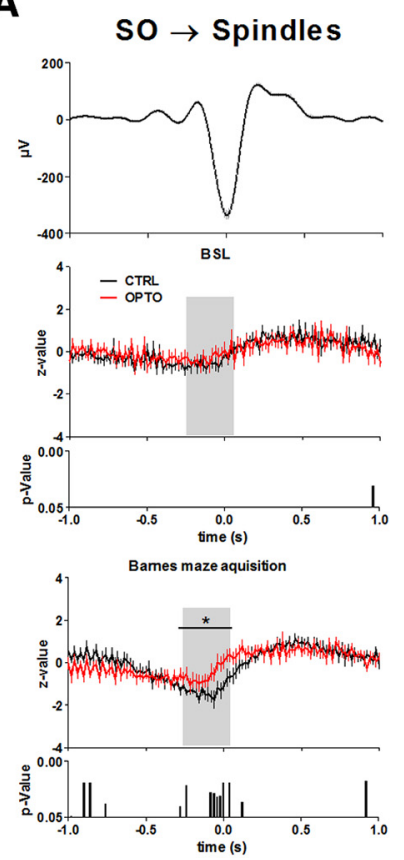

B
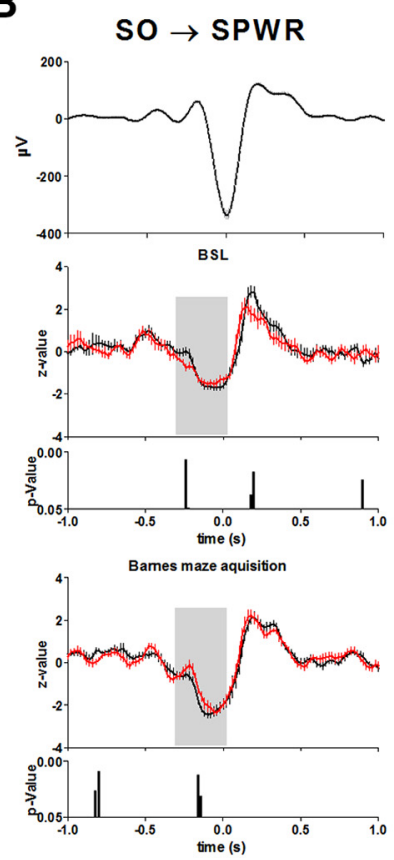

C
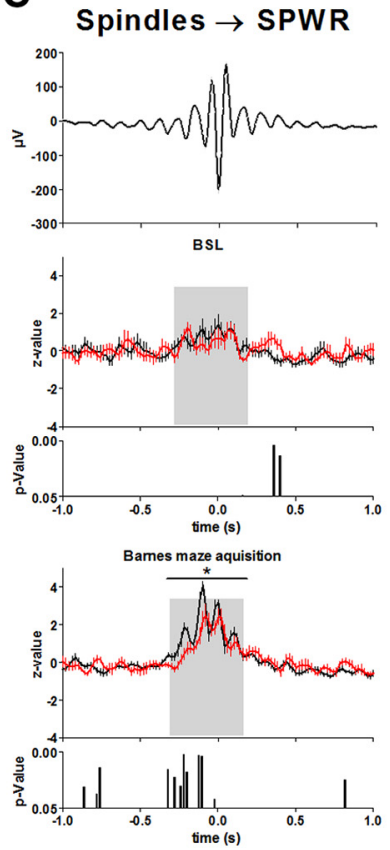

D
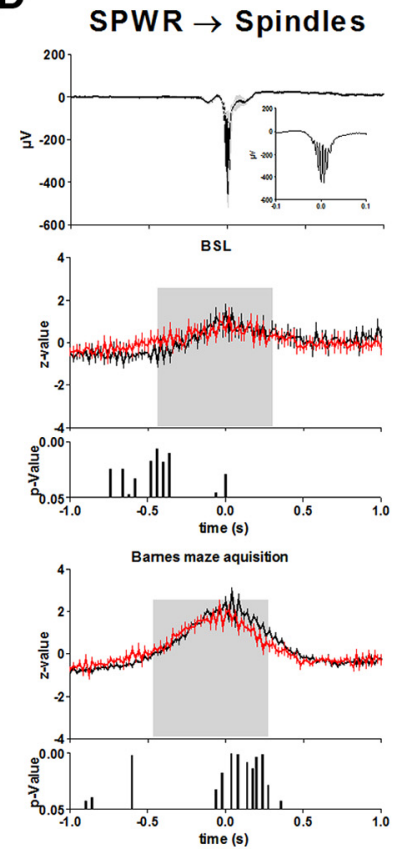

Figure 6. Event correlation histograms of SO, spindles and SPWR during BSL and Barnes maze acquisition. $\boldsymbol{A}$, Spindle activity time-locked to the negative peak of the $S 0$ ( $t=0)$. $\boldsymbol{B}$, SPWR activity time-locked to the negative peak of the $\mathrm{SO}(t=0)$. C, SPWR activity time-locked to the deepest trough of IL spindles $(t=0)$, and $\mathbf{D}$ : Spindle activity time-locked to the deepest trough of the SPWRs $(t=0)$. Top diagrams represent the mean during BSL of the CTRL of IL SO activity $(\boldsymbol{A}, \boldsymbol{B})$, IL spindles $(\boldsymbol{C})$, and dHC SPWR (D). The bottom two diagrams represent in each case the $z$-transformed time-locked activity during BSL (middle) and learning in the Barnes maze (mean across acquisition Days 1-4) with the lower bar charts representing bin-wise $p$ values (two-tailed $t$ tests) for comparisons between OPTO and CTRL. Gray shaded areas represent the time window sensitive for learning-induced modulation. $N=10$ for both groups. Error bars indicate SEM. ${ }^{*} p<0.05$.

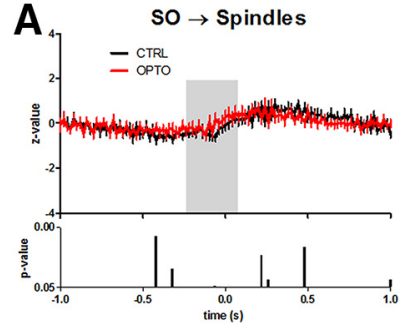

C

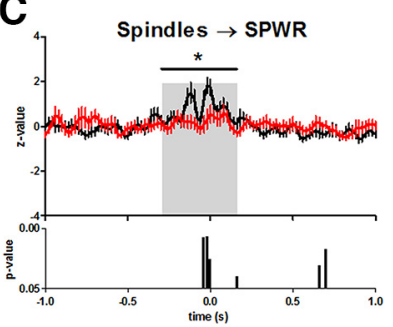

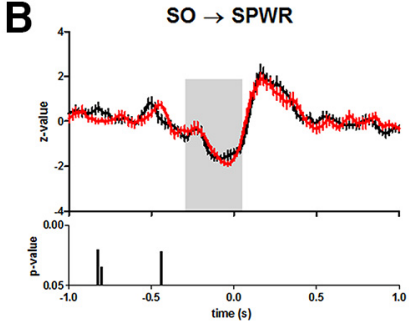

D

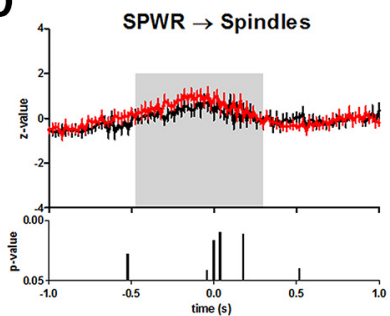

Figure 7. Event correlation histograms of SO, spindles, and SPWR during the non-learning control Experiment 2. $A$, Mean IL spindle activity time-locked to the negative peak of the IL SO $(t=0)$. B , Mean dHC SPWR activity time-locked to the negative peak of the IL SO $(t=0)$. $\boldsymbol{C}$, Mean dHC SPWR activity time-locked to the deepest trough of IL spindles $(t=0)$. D, Mean spindle activity time-locked to the deepest trough of SPWRs $(t=0)$. The bottom bar charts in $\boldsymbol{A}-\boldsymbol{D}$ represent bin-wise $p$ values (two-tailed $t$ tests) for comparisons between OPTO and CTRL conditions. Optogenetic inhibition during NREM sleep reduced the modulation of SPWR activity time-locked to IL spindles. No further modulations were observed. Gray shaded areas are identical to those in Figure 6. Within-subject design. $\boldsymbol{A}, N=19 ; \boldsymbol{B}-\boldsymbol{D}, N=16$. Error bars indicate SEM. ${ }^{*} p<0.05$.
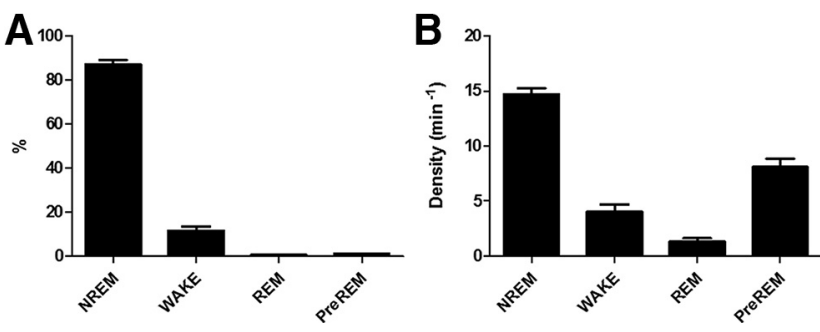

Figure 8. Distribution of laser pulses across the $3 \mathrm{~h}$ recording period in the OPTO. $\boldsymbol{A}$, Mean percentage of laser pulses of Days $1-4$ during the different sleep stages. $\boldsymbol{B}$, Mean density of laser pulses of Days $1-4$ during the different vigilant states. $N=10$. Error bars indicate SEM.

projection during NREM sleep for the relay of stored contextual information such as extra-maze cues (Royer et al., 2010; Komorowski et al., 2013) and thus subsequently lead to impaired processing in OPTO within the PFC and, putatively, in its projection sites. On the other hand, the discrepancy between the impact on learning performance and strategy use may also be related to the limited suppression of activity by our axonal inhibition procedure. A discrepancy in responsiveness of the mPFC to $\mathrm{vHC}$ stimulation between natural sleep and anesthesia cannot be excluded. Evoked field potentials cannot a priori be associated with a specific functional impact, as evidenced by distinct functional relevance of the $\mathrm{vHC}-\mathrm{mPFC}$ pathway during waking for encoding versus maintenance or retrieval of spatial cues (Spellman et al., 2015).

The surprising upregulation of $\mathrm{dHC}$ SPWR density in response to axonal silencing of hippocampal outputs to mPFC, which was most pronounced during Days 1 and 2, occurred when 


\section{A}
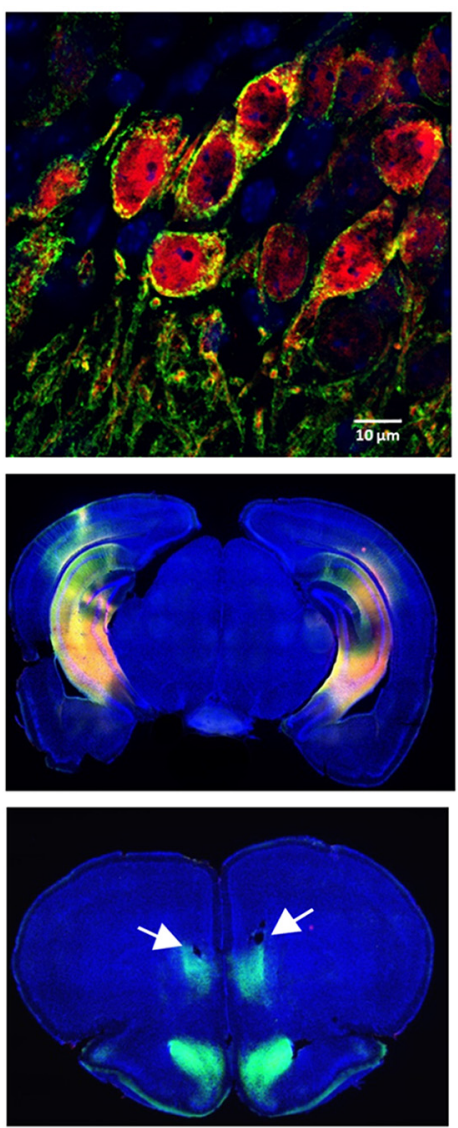

B

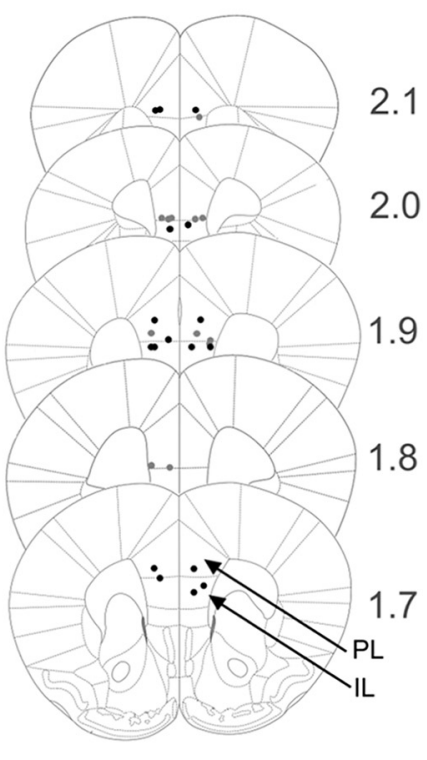

C

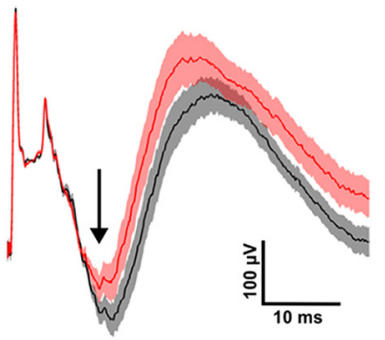

Figure 9. iChloC expression in the brain and suppression of evoked mPFC activity by optogenetic inhibition. $\boldsymbol{A}$, In representative sections, expression of iChloC in the membrane (2A tag, green) of transduced neurons (tdimer2, red) in the CA1 region of vHC. Blue, DAPI staining [scale bar (top), $10 \mu \mathrm{m}$ ] in vHC/iHC of the same animal (middle), and in the mPFC of the same animal. White arrows indicate small lesions because of optic fiber placement (bottom). $\boldsymbol{B}$, For all animals positioning of the optic fiber tips is given. Black and gray dotes indicate tips in each hemisphere of the OPTO and CTRL group, respectively. Eight of the 40 tip positions are not shown because of damaged slice tissue preparations. C, Mean vHC-evoked field potentials in mPFC with (red, OPTO trials) and without (black, CTRL trials) illumination in mPFC in an anesthetized animal. At $\sim 15-20$ ms following the electrical stimulation in VHC inputs into mPFC generate a negative deflection (black arrow) in the field potential, which is reduced in the OPTO trials. Error bars indicate SEM.

learning progress was highest. The relevance of learning for this effect was underscored by its absence in the non-learning control experiment (Experiment 2). Similar findings were reported previously (Girardeau et al., 2014; Norimoto et al., 2018). Girardeau et al. (2014) for example showed that suppression of SPWRs by short electrical pulses leads to an NMDA receptor-dependent compensatory upregulation of SPWRs following learning, but not in a non-learning control condition. The precise network mechanism of SPWR upregulation upon SPWR disturbance, and whether the present upregulation upon silencing of $\mathrm{HC}$ output seemingly not only during NREM sleep relies on a similar mechanism, still needs to be determined. Putatively, learning-induced plasticity processes and tagging of neocortical and hippocampal neurons may play a role (Lesburguères et al., 2011) and lead, together with a putative neocortical-to-HC feedback mechanism, to enhanced SPWR activity in absence of successful HC-mPFC information transfer, e.g., via the entorhinal cortex or thalamic nuclei (Vertes, 2004; Varela et al., 2014).

Whereas the density of SPWRs was increased, temporal coordination between oscillatory activities was decreased in OPTO as indicated by the reduced occurrence of SPWR nesting within spindle troughs. This pattern could also be seen in WRO-CTRL. It has been shown before that precise timing of $\mathrm{HC}-\mathrm{mPFC}$ rhythms during post-learning sleep is essential for successful memory consolidation: Enhancing the temporal coupling between SPWRs, spindles and neocortical SOs by electrical or optogenetic means was associated with improved memory consolidation in mice and humans (Maingret et al., 2016; Ladenbauer et al., 2017; Latchoumane et al., 2017). Here, a reduction in coupling of SPWR activity to spindle troughs during NREM sleep induced by silencing the $\mathrm{vHC} / \mathrm{iHC}$ output to mPFC was seen. The fact that a reduction was found during the acquisition phases and in the non-learning control experiment, underscores the relevance of the vHippmPFC pathway for the temporal linkage of SPWRs to spindles, independent of whether previous learning took place.

Modulation of sleep spindle activity time-locked to SOs was also reduced by interfering with the $\mathrm{vHC} / \mathrm{iHC}-\mathrm{mPFC}$ pathway during NREM sleep. This finding is particularly interesting because the generators of both thalamo-cortical spindles and neocortical SOs (Steriade et al., 1993) have not been associated with hippocampal input to mPFC. Furthermore, this latter effect on coupling appeared to be learning-dependent, as in our non-learning control experiment, no differences between conditions were observed. Thus, our findings strongly support that enhanced coupling between spindle activity and SOs is a marker for efficient $\mathrm{HC}-\mathrm{mPFC}$ information transfer as previously shown (Maingret et al., 2016; Weigenand et al., 2016).

Other pathways beside the monosynaptic vHC/iHC-mPFC projection were shown before to play a role for systems consolidation of hippocampus-dependent memory. These include HC-entorhinal-mPFC projections (Kitamura et al., 2017) and projections via the thalamic nucleus reuniens (Loureiro et al., 2012; Varela et al., 2014). Therefore, it could be argued that suppressing the direct $\mathrm{vHC} / \mathrm{iHC}-\mathrm{mPFC}$ projection would only moderately impair spatial memory consolidation, but not entirely prevent information transfer from $\mathrm{HC}$ to mPFC. It could be speculated that the different pathways contribute to the consolidation of different behavioral tasks or specific aspects. Unfortunately, systematic investigations on this issue are still missing.

\section{Conclusions}

Together, our results indicate that the vHC/iHC-mPFC projection contributes to sleep-dependent spatial memory consolidation, probably by shaping the temporal coordination of sleep-associated electrophysiological events, involving not only the hippocampus, but also thalamo-cortical rhythms. Furthermore, our results may contribute to the debate on the nature of information consolidated via the vHC-mPFC pathway in sleep. 
A
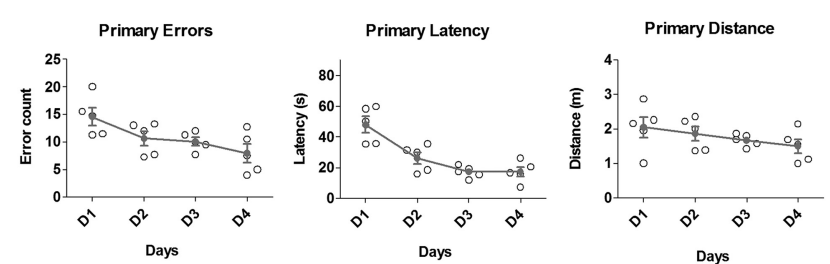

B
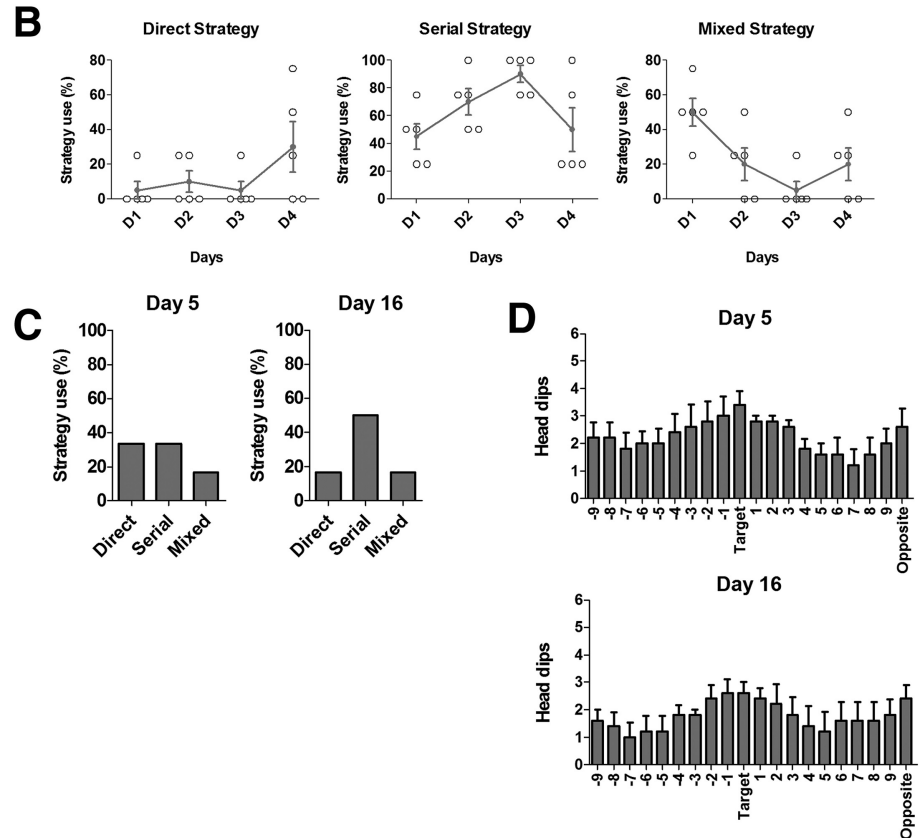

E
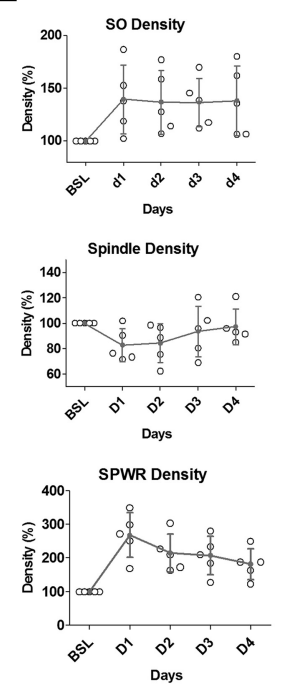

G

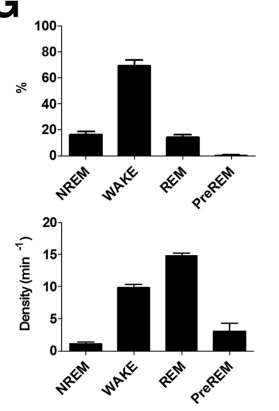

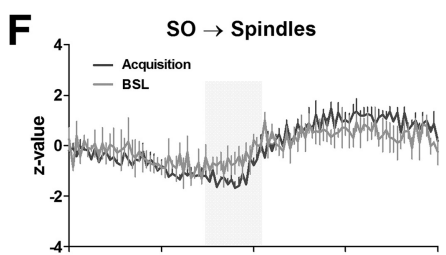
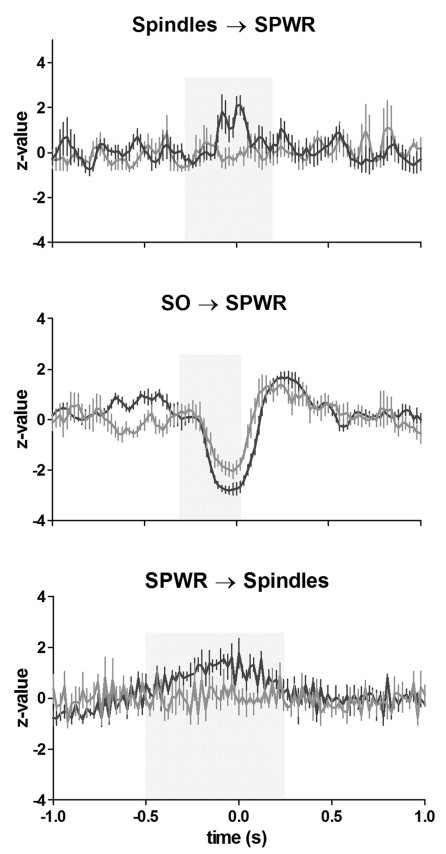

Figure 10. Optogenetic inhibition during wakefulness and REM sleep (WRO-CTRL group). $\boldsymbol{A}-\boldsymbol{D}$, Barnes maze performance. $\boldsymbol{A}$, Left to right, Time course across acquisition Days 1-4 (D1-D4) with 4 trials/d, of primary errors, primary latency, and primary distance for the WRO-CTRL. Gray symbols, Mean \pm SEM; open circles, individual animals. $\boldsymbol{B}$, Percentage of behavioral trials indicating use of direct, serial or mixed strategy for the WRO-CTRL on acquisition Days 1-4. Gray symbols, Mean \pm SEM; open circles, individual animals. C, Percentage of behavior indicating use of direct, serial, or mixed strategy for the WRO-CTRL on probe Days 5 and 16. D. Mean number of head dips into each hole on Day 5 (top) and Day 16 (bottom) for the WRO-CTRL. Error bars indicate SEM. E, SO (top), spindle (middle), and SPWR (bottom) densities in IL during post-learning NREM sleep of acquisition Days 1-4 compared with BSL. Gray symbols, Mean \pm SEM; open circles, individual animals. $\boldsymbol{F}$, Event correlation histograms of S0, spindles, and SPWR during BSL and Barnes maze acquisition for the WRO-CTRL. For detailed explanation of axes and symbols, see Figure 6. G, Distribution of laser pulses across the $3 \mathrm{~h}$ recording period for the WRO-CTRL group. Left, Mean percentage of laser pulses of Days $1-4$ during the different sleep stages. Right, Mean density of laser pulses of Days $1-4$ during the different vigilant states. Error bars indicate SEM. For all graphs, $N=5$.

\section{References}

Adhikari A, Topiwala MA, Gordon JA (2010) Synchronized activity between the ventral hippocampus and the medial prefrontal cortex during anxiety. Neuron 65:257-269.

Binder S, Berg K, Gasca F, Lafon B, Parra LC, Born J, Marshall L (2014) Transcranial slow oscillation stimulation during sleep enhances memory consolidation in rats. Brain Stimul 7:508-515.

Bontempi B, Laurent-Demir C, Destrade C, Jaffard R (1999) Timedependent reorganization of brain circuitry underlying long-term memory storage. Nature 400:671-675.

Carreno FR, Donegan JJ, Boley AM, Shah A, DeGuzman M, Frazer A, Lodge DJ (2016) Activation of a ventral hippocampus-medial prefrontal cortex pathway is both necessary and sufficient for an antidepressant response to ketamine. Mol Psychiatry 21:1298-1308.

Cenquizca LA, Swanson LW (2007) Spatial organization of direct hippocampal field CA1 axonal projections to the rest of the cerebral cortex. Brain Res Rev 56:1-26.

Cetin A, Komai S, Eliava M, Seeburg PH, Osten P (2006) Stereotaxic gene delivery in the rodent brain. Nat Protoc 1:3166-3173.

Chauvette S, Seigneur J, Timofeev I (2012) Sleep oscillations in the thalamocortical system induce long-term neuronal plasticity. Neuron 75:11051113.

Clemens Z, Fabó D, Halász P (2005) Overnight verbal memory retention correlates with the number of sleep spindles. Neuroscience 132:529-535.

Cox R, Hofman WF, Talamini LM (2012) Involvement of spindles in memory consolidation is slow wave sleep-specific. Learn Mem 19:264-267.
Diba K, Buzsáki G (2007) Forward and reverse hippocampal place-cell sequences during ripples. Nat Neurosci 10:1241-1242.

Ego-Stengel V, Wilson MA (2010) Disruption of ripple-associated hippocampal activity during rest impairs spatial learning in the rat. Hippocampus 20:1-10.

Eichenbaum H (2017) Prefrontal-hippocampal interactions in episodic memory. Nat Rev Neurosci 18:547-558.

Eschenko O, Mölle M, Born J, Sara SJ (2006) Elevated sleep spindle density after learning or after retrieval in rats. J Neurosci 26:12914-12920.

Eschenko O, Ramadan W, Mölle M, Born J, Sara SJ (2008) Sustained increase in hippocampal sharp-wave ripple activity during slow-wave sleep after learning. Learn Mem 15:222-228.

Foster DJ, Wilson MA (2006) Reverse replay of behavioural sequences in hippocampal place cells during the awake state. Nature 440:680-683.

Frankland PW, Bontempi B (2005) The organization of recent and remote memories. Nat Rev Neurosci 6:119-130.

Gais S, Mölle M, Helms K, Born J (2002) Learning-dependent increases in sleep spindle density. J Neurosci 22:6830-6834.

Girardeau G, Benchenane K, Wiener SI, Buzsáki G, Zugaro MB (2009) Selective suppression of hippocampal ripples impairs spatial memory. Nat Neurosci 12:1222-1223.

Girardeau G, Cei A, Zugaro M (2014) Learning-induced plasticity regulates hippocampal sharp wave-ripple drive. J Neurosci 34:5176-5183.

Harrison FE, Reiserer RS, Tomarken AJ, McDonald MP (2006) Spatial and nonspatial escape strategies in the barnes maze. Learn Mem 13:809-819. 
Hoover WB, Vertes RP (2007) Anatomical analysis of afferent projections to the medial prefrontal cortex in the rat. Brain Struct Funct 212:149-179.

Insausti R, Herrero MT, Witter MP (1997) Entorhinal cortex of the rat: cytoarchitectonic subdivisions and the origin and distribution of cortical efferents. Hippocampus 7:146-183.

Isomura Y, Sirota A, Ozen S, Montgomery S, Mizuseki K, Henze DA, Buzsáki $G$ (2006) Integration and segregation of activity in entorhinalhippocampal subregions by neocortical slow oscillations. Neuron $52: 871-882$.

Jadhav SP, Kemere C, German PW, Frank LM (2012) Awake hippocampal sharp-wave ripples support spatial memory. Science 336:1454-1458.

Kitamura T, Ogawa SK, Roy DS, Okuyama T, Morrissey MD, Smith LM, Redondo RL, Tonegawa S (2017) Engrams and circuits crucial for systems consolidation of a memory. Science 356:73-78.

Komorowski RW, Garcia CG, Wilson A, Hattori S, Howard MW, Eichenbaum H (2013) Ventral hippocampal neurons are shaped by experience to represent behaviorally relevant contexts. J Neurosci 33:8079-8087.

Ladenbauer J, Ladenbauer J, Külzow N, de Boor R, Avramova E, Grittner U, Flöel A (2017) Promoting sleep oscillations and their functional coupling by transcranial stimulation enhances memory consolidation in mild cognitive impairment. J Neurosci 37:7111-7124.

Laroche S, Davis S, Jay TM (2000) Plasticity at hippocampal to prefrontal cortex synapses: dual roles in working memory and consolidation. Hippocampus 10:438-446.

Latchoumane CV, Ngo HV, Born J, Shin HS (2017) Thalamic spindles promote memory formation during sleep through triple phase-locking of cortical, thalamic, and hippocampal rhythms. Neuron 95:424-435.e6.

Lesburguères E, Gobbo OL, Alaux-Cantin S, Hambucken A, Trifilieff P, Bontempi B (2011) Early tagging of cortical networks is required for the formation of enduring associative memory. Science 331:924-928.

Loureiro M, Cholvin T, Lopez J, Merienne N, Latreche A, Cosquer B, Geiger K, Kelche C, Cassel JC, Pereira de Vasconcelos A (2012) The ventral midline thalamus (reuniens and rhomboid nuclei) contributes to the persistence of spatial memory in rats. J Neurosci 32:9947-9959.

Maingret N, Girardeau G, Todorova R, Goutierre M, Zugaro M (2016) Hippocampo-cortical coupling mediates memory consolidation during sleep. Nat Neurosci 19:959-964.

Marr D (1971) Simple memory: a theory for archicortex. Philos Trans R Soc Lond B Biol Sci 262:23-81.

Marshall L, Helgadóttir H, Mölle M, Born J (2006) Boosting slow oscillations during sleep potentiates memory. Nature 444:610-613.

Maviel T, Durkin TP, Menzaghi F, Bontempi B (2004) Sites of neocortical reorganization critical for remote spatial memory. Science 305:96-99.

Mölle M, Yeshenko O, Marshall L, Sara SJ, Born J (2006) Hippocampal sharp wave-ripples linked to slow oscillations in rat slow-wave sleep. J Neurophysiol 96:62-70.

Mölle M, Eschenko O, Gais S, Sara SJ, Born J (2009) The influence of learning on sleep slow oscillations and associated spindles and ripples in humans and rats. Eur J Neurosci 29:1071-1081.

Ngo HV, Martinetz T, Born J, Mölle M (2013) Auditory closed-loop stimulation of the sleep slow oscillation enhances memory. Neuron 78 : $545-553$.

Norimoto H, Makino K, Gao M, Shikano Y, Okamoto K, Ishikawa T, Sasaki T, Hioki H, Fujisawa S, Ikegaya Y (2018) Hippocampal ripples downregulate synapses. Science 359:1524-1527.

Padilla-Coreano N, Bolkan SS, Pierce GM, Blackman DR, Hardin WD, Garcia-Garcia AL, Spellman TJ, Gordon JA (2016) Direct ventral hippocampal-prefrontal input is required for anxiety-related neural activity and behavior. Neuron 89:857-866.

Pereira de Vasconcelos A, Cassel JC (2015) The nonspecific thalamus: a place in a wedding bed for making memories last? Neurosci Biobehav Rev 54:175-196.

Peyrache A, Khamassi M, Benchenane K, Wiener SI, Battaglia FP (2009) Replay of rule-learning related neural patterns in the prefrontal cortex during sleep. Nat Neurosci 12:919-926.
Peyrache A, Battaglia FP, Destexhe A (2011) Inhibition recruitment in prefrontal cortex during sleep spindles and gating of hippocampal inputs. Proc Natl Acad Sci U S A 108:17207-17212.

Ramadan W, Eschenko O, Sara SJ (2009) Hippocampal sharp wave/ripples during sleep for consolidation of associative memory. PLoS One 4:e6697.

Rasch B, Born J (2013) About sleep's role in memory. Physiol Rev 93: 681-766.

Roux L, Hu B, Eichler R, Stark E, Buzsáki G (2017) Sharp wave ripples during learning stabilize the hippocampal spatial map. Nat Neurosci 20:845-853.

Royer S, Sirota A, Patel J, Buzsáki G (2010) Distinct representations and theta dynamics in dorsal and ventral hippocampus. J Neurosci 30: 1777-1787.

Sanders JI, Kepecs A (2014) A low-cost programmable pulse generator for physiology and behavior. Front Neuroeng 7:43.

Schabus M, Gruber G, Parapatics S, Sauter C, Klösch G, Anderer P, Klimesch W, Saletu B, Zeitlhofer J (2004) Sleep spindles and their significance for declarative memory consolidation. Sleep 27:1479-1485.

Sirota A, Csicsvari J, Buhl D, Buzsáki G (2003) Communication between neocortex and hippocampus during sleep in rodents. Proc Natl Acad Sci U S A 100:2065-2069.

Spellman T, Rigotti M, Ahmari SE, Fusi S, Gogos JA, Gordon JA (2015) Hippocampal-prefrontal input supports spatial encoding in working memory. Nature 522:309-314.

Steriade M, Nuñez A, Amzica F (1993) A novel slow ( $<1 \mathrm{~Hz}$ ) oscillation of neocortical neurons in vivo: depolarizing and hyperpolarizing components. J Neurosci 13:3252-3265.

Sunyer B, Patil S, Höger H, Lubec G (2007) Barnes maze, a useful task to assess spatial reference memory in the mice. Protocol Exchange 2007:10.

Swanson LW, Köhler C (1986) Anatomical evidence for direct projections from the entorhinal area to the entire cortical mantle in the rat. J Neurosci 6:3010-3023.

Tang W, Jadhav SP (2019) Sharp-wave ripples as a signature of hippocampal-prefrontal reactivation for memory during sleep and waking states. Neurobiol Learn Mem 160:11-20.

Thierry AM, Gioanni Y, Dégénétais E, Glowinski J (2000) Hippocampoprefrontal cortex pathway: anatomical and electrophysiological characteristics. Hippocampus 10:411-419.

Tripathi A, Schenker E, Spedding M, Jay TM (2016) The hippocampal to prefrontal cortex circuit in mice: a promising electrophysiological signature in models for psychiatric disorders. Brain Struct Funct 221: 2385-2391.

Varela C, Kumar S, Yang JY, Wilson MA (2014) Anatomical substrates for direct interactions between hippocampus, medial prefrontal cortex, and the thalamic nucleus reuniens. Brain Struct Funct 219:911-929.

Vertes RP (2004) Differential projections of the infralimbic and prelimbic cortex in the rat. Synapse 51:32-58.

Weigenand A, Mölle M, Werner F, Martinetz T, Marshall L (2016) Timing matters: open-loop stimulation does not improve overnight consolidation of word pairs in humans. Eur J Neurosci 44:2357-2368.

Wierzynski CM, Lubenov EV, Gu M, Siapas AG (2009) State-dependent spike-timing relationships between hippocampal and prefrontal circuits during sleep. Neuron 61:587-596.

Wietek J, Beltramo R, Scanziani M, Hegemann P, Oertner TG, Wiegert JS (2015) An improved chloride-conducting channelrhodopsin for lightinduced inhibition of neuronal activity in vivo. Sci Rep 5:14807.

Wilde C, Bruder R, Binder S, Marshall L, Schweikard A (2015) Closed-loop transcranial alternating current stimulation of slow oscillations. Curr Dir Biomed Eng 1:85-88.

Wilson MA, McNaughton BL (1994) Reactivation of hippocampal ensemble memories during sleep. Science 265:676-679.

Xia F, Richards BA, Tran MM, Josselyn SA, Takehara-Nishiuchi K, Frankland PW (2017) Parvalbumin-positive interneurons mediate neocorticalhippocampal interactions that are necessary for memory consolidation. eLife 6:e27868. 